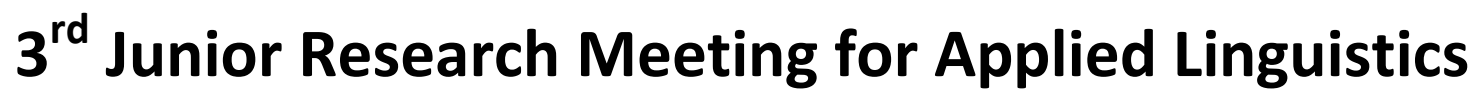 Presented by: GAL (Germany), Anéla (The Netherlands), ABLA (Belgium) and Vals-Alsa (Switzerland)
}

\author{
March $23^{\text {rd }}-25^{\text {th }}, 2011$ \\ University of Duisburg-Essen
}




\title{
Pre-Conference Workshops
}

\author{
Dr. Katja Heim \\ Qualitative Research: How to Begin the Journey
}

In this workshop we will be discussing how to begin the journey of doing qualitative research. As the message can only be that there are several ways of how to start and to structure one's research, we will be looking at different kinds of approaches and rationales. The focus will be on your work; however, i.e. a considerable part of the workshop will be spent discussing your plans.

\section{Dominik Rumlich, Ferdinand Stebner}

Statistics 101: Let data speak

To avoid the "tour de force" of an all-embracing workshop in the vast field of quantitative research, we opted for the de"light"ful version: On the basis of a small experiment in class, we are going to explore the pitfalls of quantitative data collection and then embark upon an analysis of our gathered data. In connection with our dataset and maybe one or two additional ones, we will cover the most important statistical measures in descriptive statistics and provide a quick overview of inductive statistics at the end. In class, we will use SPSS as it is one of the most widely employed software tools for data analyses in the humanities.

As you might have noticed, this path is meant to tame the often fearsome beast of quantitative research and render it more approachable and illustrative. That is to say that this workshop is meant to supply a first insight into quantitative research and possible ways of analyzing one's data without too strong a focus on the underlying mathematical operations. It is thus meant to serve as an introduction geared towards a sound understanding as well as demystification of basic statistics. 


\section{Programme Overview}

\section{Pre-Conference Workshops \\ Wednesday 23.03.2011}

\begin{tabular}{|c|c|}
\hline \multicolumn{2}{|c|}{ 13:00 Registration } \\
\hline \multicolumn{2}{|c|}{ 14:00-16:00 Pre-Conference Workshops } \\
\hline Dr. Katja Heim & $\begin{array}{l}\text { Qualitative Research: How to Begin the Journey } \\
\text { (R12 S05 H81) }\end{array}$ \\
\hline Dominik Rumlich & Statistics 101: Let data speak (R12 V05 D20) \\
\hline \multicolumn{2}{|c|}{ 16:00-16:30 Coffee Break } \\
\hline \multicolumn{2}{|c|}{ 16:30 Joint Reflection } \\
\hline
\end{tabular}

18:00 Conference Warming (EigelStein)

\section{Programme of the Sections}

Day I

Thursday 24.03.2011

\begin{tabular}{|c|c|c|}
\hline \multicolumn{3}{|c|}{$\begin{array}{c}\text { Section I } \\
\text { Language Development } \\
\text { Saskia Kersten, Petra Bosenius, Stefanie Couve de } \\
\text { Murville }\end{array}$} \\
\hline \multicolumn{3}{|c|}{ Room: R12 S05 H20 } \\
\hline \multicolumn{3}{|c|}{ 8:00 Registration } \\
\hline \multicolumn{3}{|c|}{ 9:00-10:00 Conference Opening and Keynote (Marjolijn Verspoor); Glaspavillon (R12 S0O H12) } \\
\hline \multicolumn{3}{|c|}{ 10:00-10:30 Coffee Break } \\
\hline 10:30-11:10 & Anja Binanzer & $\begin{array}{l}\text { Belebtheit im DaZ-Erwerb von } \\
\text { Genuskongruenz }\end{array}$ \\
\hline 11:10-11:50 & Ilka Peschek & $\begin{array}{l}\text { Pronominaladverbien im } \\
\text { gesprochenen Deutsch-Eine } \\
\text { gesprächsanalytische } \\
\text { Untersuchung } \\
\text { nordwestdeutscher Daten }\end{array}$ \\
\hline $11: 50-12: 30$ & Verena Wecker & $\begin{array}{l}\text { Strategien zur Bildung } \\
\text { deutscher Pluralformenbei } \\
\text { Grundschulkindern mit } \\
\text { türkischer und russischer } \\
\text { Ausgangssprache }\end{array}$ \\
\hline \multicolumn{3}{|c|}{ 12:30-13:45 Lunch Break } \\
\hline 13:45-14:25 & Dirk Janke & The Motivational Gap \\
\hline 14:25-15:05 & Amanda Post da Silveira & $\begin{array}{l}\text { Similarity: (dis)advantage for } \\
\text { English word stress acquisition } \\
\text { by Brazilian Portuguese native } \\
\text { speakers }\end{array}$ \\
\hline 15:05-15:45 & Nils Jäkel & Use and impact of language \\
\hline
\end{tabular}




\begin{tabular}{|c|l|l|}
\hline & \multicolumn{1}{|c|}{} & $\begin{array}{l}\text { learning strategies in } \\
\text { Content and Language } \\
\text { Integrated Learning (CLIL) }\end{array}$ \\
\hline 16:00-16:40 & Daniela Elsner & $\begin{array}{l}\text { Complex Constructions with } \\
\text { gucken (to look) - An analysis } \\
\text { of the most frequent matrix } \\
\text { verb in German child language }\end{array}$ \\
\hline 16:40-17:20 & $\begin{array}{l}\text { A Portuguese version of the } \\
\text { Schlichting Test for Sentence } \\
\text { Development (STSD-PT) }\end{array}$ \\
\hline $\mathbf{1 7 : 2 0 - 1 7 : 4 5}$ & Sónia Correia Vieira & \multicolumn{2}{|c|}{ Final Discussion in Section (Day1) } \\
\hline
\end{tabular}

Conference Event (Unperfekthaus)

\begin{tabular}{|c|c|c|}
\hline \multicolumn{3}{|c|}{$\begin{array}{c}\text { Section II } \\
\text { Language in Community and Media } \\
\text { Dorothee Meer, Katharina König }\end{array}$} \\
\hline \multicolumn{3}{|c|}{ Room: R12 S05 H81 } \\
\hline \multicolumn{3}{|c|}{ 8:00 Registration } \\
\hline \multicolumn{3}{|c|}{ 9:00-10:00 Conference Opening and Keynote (Marjolijn Verspoor); Glaspavillon (R12 SOO H12) } \\
\hline \multicolumn{3}{|c|}{ 10:00-10:30 Coffee Break } \\
\hline 10:30-11:10 & Julia Döring & $\begin{array}{l}\text { Moderne, Peinlichkeit" oder } \\
\text { peinliche Zeiten? Zum } \\
\text { Verhältnis von Wort, Begriff } \\
\text { und Phänomen am Beispiel von } \\
\text { „Peinlichkeit" }\end{array}$ \\
\hline 11:10-11:50 & Beatrix Fehse & $\begin{array}{l}\text { Metaphern in Text-Bild- } \\
\text { Gefügen. Verbale Metaphorik. } \\
\text { Visuelle Metaphorik. } \\
\text { Zeitmetaphern in der } \\
\text { Anzeigenwerbung und der } \\
\text { Gegenwartskunst }\end{array}$ \\
\hline 11:50-12:30 & Antonia Unger, Katja Nikitin & $\begin{array}{l}\text { Organisationskommunikation } \\
\text { im mehrsprachigen Vergleich - } \\
\text { die Krisenkommunikation von } \\
\text { BP während der Ölkatastrophe } \\
\text { im Golf von Mexiko }\end{array}$ \\
\hline \multicolumn{3}{|c|}{ 12:30-13:45 Lunch Break } \\
\hline $13: 45-14: 25$ & Julia Steube & Laienlexikografie im Internet \\
\hline 14:25-15:05 & Tanja Kaya & $\begin{array}{l}\text { Bilingual Pragmatic } \\
\text { Competence: The Use of the } \\
\text { Speech Act of Apology by } \\
\text { Turkish-German Bilingual } \\
\text { Students }\end{array}$ \\
\hline 15:05-15:45 & Juliane Mroz & $\begin{array}{l}\text { Verständlichkeit von } \\
\text { Anglizismen und Kurzwörtern in } \\
\text { Internetfachsprache und } \\
\text { Internetslang }\end{array}$ \\
\hline \multicolumn{3}{|c|}{ 15:45-16:00 Coffee Break } \\
\hline
\end{tabular}




\begin{tabular}{|c|l|l|}
\hline 16:00-16:40 & Katharina König & $\begin{array}{l}\text { Indirekte Formulierungen in } \\
\text { narrativen Interviews }\end{array}$ \\
\hline $\mathbf{1 6 : 4 0 - 1 7 : 2 0}$ & Eva Zumhasch & $\begin{array}{l}\text { The Use of English in Hong } \\
\text { Kong Rap: An Example from } \\
\text { 24Herbs }\end{array}$ \\
\hline $\mathbf{1 7 : 2 0 - 1 7 : 4 5}$ & \multicolumn{2}{|c|}{ Final Discussion in Section (Day1) } \\
\hline
\end{tabular}

Conference Event (Unperfekthaus)

\begin{tabular}{|c|c|c|}
\hline \multicolumn{3}{|c|}{$\begin{array}{c}\text { Section III } \\
\text { Language in Education } \\
\text { Martin Luginbühl }\end{array}$} \\
\hline \multicolumn{3}{|c|}{ Room: R12 V05 D20 } \\
\hline \multicolumn{3}{|c|}{ 8:00 Registration } \\
\hline \multicolumn{3}{|c|}{ 9:00-10:00 Conference Opening and Keynote (Marjolijn Verspoor); Glaspavillon (R12 S0O H12) } \\
\hline \multicolumn{3}{|c|}{ 10:00-10:30 Coffee Break } \\
\hline 10:30-11:10 & $\begin{array}{l}\text { Stefanie Couve de } \\
\text { Murville }\end{array}$ & $\begin{array}{l}\text { Wie erfolgreich ist bilingualer } \\
\text { Sachfachunterricht wirklich? - } \\
\text { Lernstandserhebungen in einer } \\
\text { immersiven Grundschule }\end{array}$ \\
\hline $11: 10-11: 50$ & Emilie Magnat & $\begin{array}{l}\text { A multisensory approach of } \\
\text { learning English L2 to work in } \\
\text { tomorrow world }\end{array}$ \\
\hline $11: 50-12: 30$ & Dr. Marta Janachowska-Budych & $\begin{array}{l}\text { Literatur und das kulturelle } \\
\text { Gedächtnis - } \\
\text { Forschungsbericht und } \\
\text { Didaktisierungsmöglichkeiten }\end{array}$ \\
\hline \multicolumn{3}{|c|}{ 12:30-13:45 Lunch Break } \\
\hline $13: 45-14: 25$ & Joanna Kic-Drgas & $\begin{array}{l}\text { Zwischen Instruktion und } \\
\text { Konstruktion- neue Perspektive } \\
\text { für den } \\
\text { Fremdsprachenunterricht im } \\
\text { Seniorenalter }\end{array}$ \\
\hline $14: 25-15: 05$ & Dominik Rumlich & $\begin{array}{l}\text { Learners' performance in } \\
\text { written English: Do CLIL } \\
\text { students achieve higher levels } \\
\text { of accuracy than their peers in } \\
\text { 'regular' classes? }\end{array}$ \\
\hline $15: 05-15: 45$ & Verena Möller & $\begin{array}{l}\text { English Passive Constructions in } \\
\text { the Interlanguage of German } \\
\text { Learners }\end{array}$ \\
\hline \multicolumn{3}{|c|}{ 15:45-16:00 Coffee Break } \\
\hline $16: 00-16: 40$ & Annelien De Geest & $\begin{array}{l}\text { Entering university: a process of } \\
\text { acculturation }\end{array}$ \\
\hline $16: 40-17: 20$ & Agnieszka Świrko & $\begin{array}{l}\text { Die blinden Schüler und der } \\
\text { Fremdsprachenunterricht }\end{array}$ \\
\hline $17: 20-17: 45$ & \multicolumn{2}{|c|}{ Final Discussion in Section (Day1) } \\
\hline
\end{tabular}




\begin{tabular}{|c|c|c|}
\hline \multicolumn{3}{|c|}{$\begin{array}{c}\text { Section IV } \\
\text { Language in Professional Settings } \\
\text { Kris van de Poel }\end{array}$} \\
\hline \multicolumn{3}{|c|}{ Room: R12 V05 D81 } \\
\hline \multicolumn{3}{|c|}{ 8:00 Registration } \\
\hline \multicolumn{3}{|c|}{ 9:00-10:00 Conference Opening and Keynote (Marjolijn Verspoor); Glaspavillon (R12 S00 H12) } \\
\hline \multicolumn{3}{|c|}{ 10:00-10:30 Coffee Break } \\
\hline 10:30-11:10 & Petra Klimant & $\begin{array}{l}\text { Mentale Erschöpfung und } \\
\text { Übersetzungsqualität }\end{array}$ \\
\hline 11:10-11:50 & Andrea Hunziker Heeb & $\begin{array}{l}\text { A window onto the translator's } \\
\text { mind: how process research } \\
\text { helps shed light on problem } \\
\text { awareness }\end{array}$ \\
\hline 11:50-12:30 & Dr. Gabriela Gorąca & $\begin{array}{l}\text { Linguisten und ihre } \\
\text { Kompetenzen in linguistischen } \\
\text { Berufen }\end{array}$ \\
\hline \multicolumn{3}{|c|}{ 12:30-13:45 Lunch Break } \\
\hline 13:45-14:25 & Julia Spanke & $\begin{array}{l}\text { Verständigungsprobleme. Eine } \\
\text { empirische Analyse am Beispiel } \\
\text { der } \\
\text { industriellen } \\
\text { Prozessmodellierung }\end{array}$ \\
\hline 14:25-15:05 & Ingmar Rothe & $\begin{array}{l}\text { Strikt neben dem Leitfaden. } \\
\text { Analysen skriptbasierter } \\
\text { Telefonie. }\end{array}$ \\
\hline 15:05-15:45 & Milan Mihajlovic & $\begin{array}{l}\text { Jugend debattiert- a pragma- } \\
\text { dialectical study }\end{array}$ \\
\hline \multicolumn{3}{|c|}{ 15:45-16:00 Coffee Break } \\
\hline 16:00-16:40 & Gabriella Carobbio & $\begin{array}{l}\text { "Wie Sie sehen können..." - } \\
\text { Einwirkungen multimedialer } \\
\text { Präsentationen auf Vorträge }\end{array}$ \\
\hline 16:40-17:20 & Jan Gerwinski & $\begin{array}{l}\text { Medienvermittelte } \\
\text { Ortserkundung in multilokalen } \\
\text { und multimodalen } \\
\text { Interaktionsräumen - am } \\
\text { Beispiel von } \\
\text { Feuerwehreinsatzübungen }\end{array}$ \\
\hline
\end{tabular}

\section{Conference Event (Unperfekthaus)}




\section{Programme of the Sections}

Day II

Friday 25.03.2011

\begin{tabular}{|c|c|c|}
\hline \multicolumn{3}{|c|}{$\begin{array}{c}\text { Section I } \\
\text { Language Development } \\
\text { Saskia Kersten, Petra Bosenius, Stefanie Couve de } \\
\text { Murville }\end{array}$} \\
\hline \multicolumn{3}{|c|}{ Room: R12 S05 H20 } \\
\hline \multicolumn{3}{|c|}{ 8:00 Registration } \\
\hline 09:00-09:40 & Nicole Riechert & $\begin{array}{l}\text { Die (neuen) Stimmen der } \\
\text { Jugend: Über die Signifikanz } \\
\text { der deutschen Rapmusik }\end{array}$ \\
\hline 09:40-10:20 & Christian Ludwig & $\begin{array}{l}\text { What Comics can do: the Use of } \\
\text { Graphic Fiction in SLA- } \\
\text { Processes and the Development } \\
\text { of Visual Literacy }\end{array}$ \\
\hline 10:20-11:00 & Jana Gamper & $\begin{array}{l}\text { Der Junge zeigt den Polizist die } \\
\text { Zunge» - Kasusmarkierungen in } \\
\text { Texten von DaZ-Lernern }\end{array}$ \\
\hline \multicolumn{3}{|c|}{ 11:00-11:30 Coffee Break } \\
\hline \multicolumn{3}{|c|}{ 11:30-12:15 Keynote (Gisela Brünner); Glaspavillon (R12 S00 H12) } \\
\hline \multicolumn{3}{|c|}{ 12:15-13:30 Lunch } \\
\hline 13:30-14:10 & Margit Hempel & $\begin{array}{l}\text { Englisch Lernen als Kontinuum } \\
\text { - Entwicklung von produktiven } \\
\text { Fähigkeiten und Fertigkeiten } \\
\text { beim Übergang von der } \\
\text { Primarstufe in die } \\
\text { Sekundarstufe }\end{array}$ \\
\hline 14:10-14:50 & Hana Smiskova & $\begin{array}{l}\text { Conventionalized sequences in } \\
\text { L2 development }\end{array}$ \\
\hline 14:50-15:30 & Dorina Veldhuis, Ad Backus & $\begin{array}{l}\text { Chunks for segmentation and } \\
\text { processing }\end{array}$ \\
\hline $15: 30-15: 40$ & \multicolumn{2}{|c|}{ Final Discussion in Section (Day2) } \\
\hline \multicolumn{3}{|c|}{ 15:40-16:00 Coffee Break } \\
\hline & ing of Conference; Glaspai & R12 SOO H12) \\
\hline
\end{tabular}

\begin{tabular}{|c|c|c|}
\hline \multicolumn{3}{|c|}{$\begin{array}{c}\text { Section II } \\
\text { Language in Community and Media } \\
\text { Dorothee Meer, Katharina König }\end{array}$} \\
\hline \multicolumn{3}{|c|}{ Room: R12 S05 H81 } \\
\hline \multicolumn{3}{|c|}{ 8:00 Registration } \\
\hline 09:00-09:40 & Jude Ssempuuma & $\begin{array}{l}\text { The Role of English in Ugandan } \\
\text { Political Debates: Hedges and } \\
\text { Rhetoric Questions in Political } \\
\text { Campaigns in Kampala } \\
\end{array}$ \\
\hline $09: 40-10: 20$ & Anna Tkachenko & „ich bin ein stolzer mixer \\
\hline
\end{tabular}




\begin{tabular}{|c|c|c|}
\hline & & $\begin{array}{l}\text { zwischen Russe und } \\
\text { Deutscher": } \\
\text { Versprachlichungsstrategien } \\
\text { russischer Migranten im Chat }\end{array}$ \\
\hline 10:20-11:00 & Julia Wrede & $\begin{array}{l}\text { Bedeutungs-(Re)Konstruktion } \\
\text { durch den Sprachgebrauch: } \\
\text { Eine korpuslinguistische } \\
\text { Untersuchung kognitiver } \\
\text { Phänomene und semantischer } \\
\text { Konsequenzen }\end{array}$ \\
\hline \multicolumn{3}{|c|}{ 11:00-11:30 Coffee Break } \\
\hline \multicolumn{3}{|c|}{ 11:30-12:15 Keynote (Gisela Brünner); Glaspavillon (R12 S0O H12) } \\
\hline \multicolumn{3}{|c|}{$12: 15-13: 30$ Lunch } \\
\hline $13: 30-14: 40$ & $\begin{array}{l}\text { Julia Richling, Bianka Trevisan, } \\
\text { Eugenie Giesbrecht, Michael } \\
\text { Beißwenger }\end{array}$ & $\begin{array}{l}\text { Korpusgestützte Analyse } \\
\text { sprachlicher Besonderheiten in } \\
\text { der internetbasierten } \\
\text { Kommunikation: } \\
\text { Brückenschläge zwischen } \\
\text { Angewandter Linguistik und } \\
\text { Maschineller } \\
\text { Sprachverarbeitung }\end{array}$ \\
\hline $14: 40-15: 20$ & Juliane Gall & $\begin{array}{l}\text { Sprache im Alter - Der Einfluss } \\
\text { sozialer Faktoren auf } \\
\text { Sprachliche Veränderungen bei } \\
\text { älteren Menschen }\end{array}$ \\
\hline $15: 20-15: 40$ & \multicolumn{2}{|c|}{ Final Discussion in Section (Day2) } \\
\hline \multicolumn{3}{|c|}{ 15:40-16:00 Coffee Break } \\
\hline
\end{tabular}

\begin{tabular}{|c|c|c|}
\hline \multicolumn{3}{|c|}{$\begin{array}{c}\text { Section III } \\
\text { Language in Education } \\
\text { Martin Luginbühl } \\
\end{array}$} \\
\hline \multicolumn{3}{|c|}{ Room: R12 V05 D20 } \\
\hline \multicolumn{3}{|c|}{ 8:00 Registration } \\
\hline 09:00-09:40 & Jacqueline Thissen & $\begin{array}{l}\text { Kleine Wörtchen haben es in } \\
\text { sich! Sind Partikeln im engeren } \\
\text { Sinne doch ein Thema für die } \\
\text { Schule? }\end{array}$ \\
\hline 09:40-10:20 & Jan Jorissen & $\begin{array}{l}\text { The Effect of Writing } \\
\text { Assignments in an Academic } \\
\text { Writing Programme }\end{array}$ \\
\hline 10:20-11:00 & Carola Strobl & $\begin{array}{l}\text { Summary writing from aural } \\
\text { input in a foreign language: } \\
\text { How can this complex task be } \\
\text { supported by an online learning } \\
\text { module? }\end{array}$ \\
\hline \multicolumn{3}{|c|}{ 11:00-11:30 Coffee Break } \\
\hline \multicolumn{3}{|c|}{ 11:30-12:15 Keynote (Gisela Brünner); Glaspavillon (R12 S00 H12) } \\
\hline \multicolumn{3}{|c|}{ 12:15-13:30 Lunch } \\
\hline 13:30-14:10 & Deogratias Nizonkiza & $\begin{array}{l}\text { Relationship between lexical } \\
\text { competence, collocational } \\
\text { competence, and second } \\
\text { language proficiency: The case }\end{array}$ \\
\hline
\end{tabular}




\begin{tabular}{|c|c|c|}
\hline & & of Belgian English majors \\
\hline $14: 10-14: 50$ & $\begin{array}{l}\text { Jelena Danilović, Ivana Zorica, } \\
\text { Branimir Jeremić }\end{array}$ & $\begin{array}{l}\text { Serbian, English and German in } \\
\text { interaction: } \\
\text { A study of cognates and } \\
\text { German L3 learners in Serbia }\end{array}$ \\
\hline 14:50-15:30 & Dieuwerke Rutgers & $\begin{array}{l}\text { Metalinguistic Development in } \\
\text { Context: Learning German as a } \\
\text { Third Language within a Dutch- } \\
\text { English Bilingual Education } \\
\text { > Programme and a regular } \\
\text { programme in the Netherlands }\end{array}$ \\
\hline $15: 30-15: 40$ & \multicolumn{2}{|c|}{ Final Discussion in Section (Day2) } \\
\hline \multicolumn{3}{|c|}{ 15:40-16:00 Coffee Break } \\
\hline \multicolumn{3}{|c|}{ 16:00-16:45 Closing of Conference; Glaspavillon (R12 SOO H12) } \\
\hline
\end{tabular}

\begin{tabular}{|c|c|c|}
\hline \multicolumn{3}{|c|}{$\begin{array}{c}\text { Section IV } \\
\text { Language in Professional Settings } \\
\text { Kris van de Poel }\end{array}$} \\
\hline \multicolumn{3}{|c|}{ Room: R12 V05 D81 } \\
\hline \multicolumn{3}{|c|}{ 8:00 Registration } \\
\hline 09:00-09:40 & To be announced soon & To be announced soon \\
\hline 09:40-10:20 & $\begin{array}{l}\text { Gregor Geiermann, Friederike } \\
\text { Sell }\end{array}$ & $\begin{array}{l}\text { How to greet a professor?": } \\
\text { identifying emerging e-mail } \\
\text { etiquette in professor-student } \\
\text { communication }\end{array}$ \\
\hline 10:20-11:00 & Inge Blockmans & $\begin{array}{l}\text { Accommodation in Special } \\
\text { Needs Communication: } \\
\text { Disabled Student's. Perception } \\
\text { of Communication about } \\
\text { Personal Functional Problems }\end{array}$ \\
\hline \multicolumn{3}{|c|}{ 11:00-11:30 Coffee Break } \\
\hline \multicolumn{3}{|c|}{ 11:30-12:15 Keynote (Gisela Brünner); Glaspavillon (R12 S00 H12) } \\
\hline \multicolumn{3}{|c|}{ 12:15-13:30 Lunch } \\
\hline 13:30-14:10 & Almut Schön & $\begin{array}{l}\text { Arzt-Patienten-Interaktion1 und } \\
\text { MS-NMS-Kommunikation: } \\
\text { Gespräche ausländischer Ärzte } \\
\text { mit deutschen Patienten aus } \\
\text { diskursanalytischer Sicht }\end{array}$ \\
\hline 14:10-14:50 & Donata Lisaité & $\begin{array}{l}\text { Mobile Medical Professionals: } \\
\text { Transcultural Communication } \\
\text { Problems on the Workfloor }\end{array}$ \\
\hline 14:50-15:30 & Verena Warda & $\begin{array}{l}\text { The Motivation of Teachers of } \\
\text { EFL in Teacher Training in NRW }\end{array}$ \\
\hline 15:30-15:40 & \multicolumn{2}{|c|}{ Final Discussion in Section (Day2) } \\
\hline \multicolumn{3}{|c|}{ 15:40-16:00 Coffee Break } \\
\hline 16:0 & sing of Conference; Glaspavil & (R12 S00 H12) \\
\hline
\end{tabular}




\section{Abstracts}

\section{Section 1 Language Development}

\section{Anja Binanzer, M.A.}

Belebtheit im DaZ-Erwerb von Genuskongruenz

Im Zusammenhang mit der "Animacy-Hierarchy" Human > Animal > Inanimate nach SILVERSTEIN (1976) wurden verschiedene grammatische Kategorien untersucht, worunter auch das semantische Genus (DAHL 2000) fällt. Im einer sprachvergleichenden Studie CORBETTS (1991) wird festgestellt, dass Genus entweder als arbiträr oder semantisch vergeben erscheint, wobei bei der semantischen Vergabe immer das Merkmal Belebtheit eine Rolle spielt. Zwischen Genuszuweisung und Belebtheit ist demnach ein Zusammenhang zu konstatieren.

In meinem Beitrag frage ich danach, ob es diesen Zusammenhang über die Genuszuweisung hinaus auch in der Genuskongruenz gibt. Ich untersuche, ob die Belebtheit bzw. Unbelebtheit von Nomen Einfluss auf den Erwerb der Genuskongruenz bei Grundschulkindern mit Türkisch und Russisch als Erstsprache nimmt. Das Genus wird im Deutschen in den meisten Fällen nicht am Nomen selbst markiert (WEGENER 1995, WEGERA 1997, MENZEL 2004), sondern zeigt sich an allen morphosyntaktisch vom Nomen kontrollierten Artikeln und Attributen innerhalb der NP sowie an substantivischen Pronomen außerhalb der NP (WERNER 1975). Damit fungiert das Nomen als Source, die von inm kontrollierten sprachlichen Elemente als Targets (CORBETT 2003), auf die das Genus des Nomens übertragen werden muss. Ich folge der Hypothese, dass Genuskongruenz bei den Targets der Nomen mit dem Merkmal [+belebt] eher zielsprachlich korrekt ausgebildet wird als bei Targets von Nomen mit dem Merkmal [- belebt].

Die vorzustellenden Daten setzen sich einerseits aus natürlichsprachlichem, andererseits aus elizitiertem schriftlichen Sprachmaterial von Grundschulkindern mit türkischer und russischer Erstsprache zusammen. Die natürlichsprachlichen Daten wurden mittels Bildimpuls mit belebten und unbelebten Sources erhoben, wozu die Kinder eine Geschichte schreiben sollten. Die gleichen Nomen wurden einen Monat später in einem MultipleChoice-Test wiederverwendet. Die Kinder mussten den Nomen selbst das Genus zuweisen (definiter/indefiniter Artikel) und in den Folgesätzen jeweils eines von drei möglichen vorgegebenen Targets (attributives Adjektiv, Perso-nal-, Possessiv- und Relativpronomen) kongruent zum Nomen wählen.

\section{Literatur}

CORBETT, Greville (2006): Agreement. Cambridge: Cambridge University Press.

- (2003): „The Agreement Hierarchy”. In: Katamba, Francis X. (Hrsg.): Morphology: Critical concepts in linguistics: IV: Morphology and Syntax. London: Routledge, 48-70 [Nachdruck von 1979, in: Journal of linguistics, 15, 203-224].

- (1991): Gender. Cambridge: Cambridge University Press. 
DAHL, Östen (2000): Animacy and the notion of semantic gender. In: Barbara Unterbeck/Matti Rissanen/Terttu Nevalainen/Mirja Saari (Hrsg.): Gender in grammar and cognition. Berlin; New York: de Gruyter, 99-115.

MENZEL, Barbara (2004): Genuszuweisung im DaF-Erwerb: psycholinguistische Prozesse und didaktische Implikationen. Berlin: Weißensee.

SILVERSTEIN, Michael (1976): Hierarchy of features and ergativity. In: Robert M. W. Dixon (Hrsg.): Grammatical categories in Australian languages. Australian Institute of Aboriginal Studies, Canberra, 112-171.

WEGENER, Heide (1995): „Das Genus im DaZ-Erwerb. Beobachtungen an Kindern aus Polen, Rußland und der Türkei." In: Handwerker, B. (Hrsg.): Fremde Sprache Deutsch. Tübingen: Narr, 1-24.

WEGERA, Klaus-Peter (1997): Das Genus. Ein Beitrag zur Didaktik des DaF-Unterrichts. München: ludicum.

WERNER, Otmar (1975): „Zum Genus im Deutschen“. In: Deutsche Sprache, 1/75, 35-48.

\section{Ilka Peschek, M.A.}

Pronominaladverbien im gesprochenen Deutsch-Eine gesprächsanalytische Untersuchung nordwestdeutscher Daten

Gegenstand des Projekts ist ein aktuelles Phänomen der Syntax des gesprochenen Deutsch: die Verwendung verschiedener Realisierungsweisen von Pronominaladverbien. Neben der als standardsprachlich kodifizierten Norm sind im alltäglichen Sprachgebrauch vielfältige Realisierungen von Pronominaladverbien anzutreffen, z. B. wodrauf, da ... für etc.

Ziel der Arbeit ist es, die kommunikative Verwendung der unterschiedlichen Formen zu erforschen und zu prüfen, inwieweit ihre Beschreibung im Rahmen der Construction Grammar dazu beitragen kann, alle Realisierungsweisen zusammenhängend in einem als „psychologisch realistisch“ (Deppermann, A. (2006): Construction Grammar - Eine Grammatik für die Interaktion? In: Deppermann, A./Fiehler, R./Spranz-Fogasy, T. (Hg.): Grammatik und Interaktion. Radolfzell: Verlag für Gesprächsforschung, Seite 43-65) geltenden Modell zu erfassen. Im Zentrum der Untersuchung des interaktiven Gebrauchs der Pronominaladverbien stehen unter anderem die Fragen, ob verschiedene Realisierungsweisen als Ressourcen zur Kontextualisierung von Nähe/Informalität gebraucht werden und ob eine (formelhafte) Verfestigung bestimmter Formen - z. B. mit bestimmten Verben - zu beobachten ist. Der Schwerpunkt der Analyse liegt auf den Distanzkonstruktionen: „Da kommen wir übrigens gleich noch zu“. Dazu werden freie gesprochene Interaktionen von SprecherInnen aus Münster (Westf.), Bochum, Dortmund, Duisburg, Hannover, Hamburg, Kiel und Bremen nach dem Gesprächsanalytischen Transkriptionssystem (GAT 2) (Selting, M./Auer, P. et al. (2009): Gesprächsanalytisches Transkriptionssystem 2 (GAT2). In: Gesprächsforschung - Online-Zeitschrift zur verbalen Interaktion (10/2009), Seite 353-402 (www.gespraechsforschung-ozs.de) erfasst und auf Grundlage gesprächsanalytischer Methodik und der Gesprochene-Sprache-Forschung umfassend ausgewertet. Herausgearbeitet werden u. a. die Sprecherrollen, eine mögliche gattungsspezifische Verwendung, die sequentielle Positionierung der PronominaladverbKonstruktionen, ihre Syntax und Topologie, die Interaktionsmodalität/Gesprächsstil, Semantik und Prosodie sowie mögliche funktional-pragmatische Aspekte (Informationsstrukturierung, phorisch-deiktischer Gebrauch). Die gesprächsanalytischen Ergebnisse werden anschließend im Hinblick auf restringierte oder präferierte 
Verwendungskontexte gedeutet. Wann greifen welche SprecherInnen auf welche Konstruktionen zurück?

Die empirischen Ergebnisse werden anschließend an den theoretischen Rahmen der Construction Grammar rückgebunden. In diesem Zusammenhang ist vor allem zu klären, ob z. B. die formelhafte Verfestigung oder funktionale Differenzierung zu einer konstruktionseigenen Semantik bestimmter Pronominaladverb-Konstruktionen führt, welche eine Betrachtung der Formen als eigene Constructions zulässt, und in welchem Zusammenhang die diskontinuierlichen Pronominaladverbien mit ähnlichen Konstruktionen, wie z. B. Partikelverbklammern und ,da-Konstruktionen', stehen.

\section{Verena Wecker, M.A.}

Strategien zur Bildung deutscher Pluralformenbei Grundschulkindern mit türkischer und russischer Ausgangssprache

Die deutsche Pluralbildung ist aus zwei Gründen komplex und damit schwer zu erlernen: Zum einen gibt es mehrere Markierungen für den Plural, zum anderen folgt die Zuweisung der Pluralmarkierungen zu den Nomen keinen eindeutigen Regeln. In verschiedenen Analysen des deutschen Numerussystems (Augst 1979, Köpcke 1993, Mugdan 1977, Wegener 2008) wurde deutlich, dass die Distribution der Pluralmarkierungen über die Nomen vor allem durch Genus und Wortauslaut des Nomens reguliert wird. Dabei gelten jedoch eher Tendenzen als eindeutige Regularitäten. Im monolingualen Deutscherwerb erwerben Kinder das komplexe System der Pluralbildung größtenteils bis zum vollendeten vierten Lebensjahr (vgl. Szagun 2006: 86ff.).Wie erwerben aber Grundschulkinder mit türkischer und russischer Ausgangssprache dieses komplexe System? Während es im Türkischen lediglich eine Pluralmarkierung und eine phonologisch bedingte Variante gibt, das Numerussystem im Türkischen also von rein phonologischen Prinzipien gesteuert wird, wird das russische Numerussystem mit ebenfalls mehreren Pluralmarkierungen von morphologischen Prinzipien wie Genus- oder Deklinationsklassenzugehörigkeit bestimmt.

\section{Amanda Post da Silveira, M.A.}

Similarity: (dis)advantage for English word stress acquisition by Brazilian Portuguese native speakers

This paper presents a phonetic-phonological study on a corpus of non-suffixed and suffixed English words produced by adult learners whose native language is Brazilian Portuguese (henceforth BP). Taking into account that both English and Portuguese have predominant penultimate stress. This may or may not be an advantage for a BP learner of English. Pitch movement and intensity seem to be reliable correlates of stress in both languages, but syllable duration, especially vowel quantity, seems to be a challenge for BP learners of English stress. Since there are indications that learners approximate their productions in the foreign language to categories existent in their native language, such approximation is possibly fostered be an interlanguage system in which markedness constraints related to syllable weight and structure and foot building from the L1 are highly ranked and faithfulness constraints related to (i) vowel length and (ii) stressed vowel marked in the L2 input are low ranked. The stabilization of the system in this interlanguage stage has as a consequence the fossilization of stress acquisition. It can be evidenced by high percentages of penultimate stress assignment whether correct or wrong. This would indicate that the 
system producing the 'correct' productions is not the target language system. Our results bear out that the similarity evidenced in both native and target languages with regard to word stress is only superficial, since distinct systems generate them. The similarity seems to be based on lexical frequency. It presents a challenge to correct acquisition of the English penultimate stress pattern by BP learners.

\section{Nils Jäkl}

Use and impact of language learning strategies in Content and Language Integrated Learning (CLIL)

\section{Abstract will follow soon.}

\section{Daniela Elsner}

Complex Constructions with gucken (to look) - An analysis of the most frequent matrix verb in German child language

Until today there are only two studies that look at how German speaking children acquire complex constructions, namely subordinated clauses. Rothweiler (1993) on the one hand follows a generative approach and concludes that the fixation of a head parameter is responsible for the acquisition of the subordinated clause structure. This idea has been rejected because of the data available on CHILDES (cf. Brandt 2010, Elsner in press). Brandt (2010) on the other hand working within a cognitive-functional framework investigates the development of word order in subordinated clauses. She reasons that the position of the verb is dependent on the form of the matrix clause and the matrix verb.

Brandt (2010), Rothweiler (1993) and Elsner (in press) found in their data that the most frequent matrix verbs that the children produce are identical. Moreover children begin with only a small set of matrix verbs, which is extended more and more. The use of the perception verb gucken (to look) is disproportionately high. It is striking that most of the children's matrix clauses have the form of a simple imperative guck/guck mal (look). Analyzing CHILDES data the aim of my presentation is to take a close look the complex constructions with gucken. In doing so I will try to outline the development from simple to complex structures as well as to give a syntactic description of these structures. Diessel (2004) and Diessel/Tomasello (2001) were able to show that English speaking children started with very formulaic matrix clauses that developed into a more flexible use of the matrix verbs. The question arises whether this process can also be seen in the German data.

\section{References:}

Brandt, S., Lieven, E., \& Tomasello, M. (2010). Development of word order in German complement-clause constructions: Effects of input frequencies, lexical items, and discourse function. Language 86(3), 583-610.

Diessel, H. (2004). The Acquisition of Complex Sentences. Cambridge: Cambridge University Press.

Diessel, H., Tomasello, M. (2001). The acquisition of finite complement clauses in English: A usage based approach to the development of grammatical constructions. Cognitive Linguistics 12, 97-141.

Elsner, D. (in press). Guck mal, ich mache - Frühe Nebensatzkonstruktionen bei deutschsprachigen Kindern. 
Rothweiler, M. (1993). Der Erwerb von Nebensätzen im Deutschen. Eine Pilotstudie. Tübingen: Niemeyer.

Data:

http://childes.psy.cmu.edu/

\section{Sónia Correia Vieira}

A Portuguese version of the Schlichting Test for Sentence Development (STSD-PT)

In Portugal, there is no standardized language production test focusing on syntax. A syntactic assessment instrument is essential in identifying children with Specific Language Impairment (SLI), especially the ones for whom the acquisition of grammar is more problematic. The method often used by the speech therapists is the assessment of spontaneous speech. Although very rich in content, it is difficult to rate and it is time-consuming. We are developing a Portuguese syntactic production test (STSD-PT) based on a Dutch sub-test for sentence development (Schlichting et al., 1995). Our research question was to investigate if the Portuguese standardization of the Schlichting test for sentence development is a valid and reliable instrument for syntactic assessment in pre-school children. The main elicitation technique used in the test is imitation. This elicitation procedure is designed in a way to involve children in a game (functional context) in which it makes sense to elicit utterances (communicative purpose).

Out of the 40 syntactic structures of the original Dutch test, 24 were maintained in the Portuguese version and for 16 of the items new syntactic structures were created for the STSD-PT. The choice of these new structures was not straightforward since only recently more studies emerge relative to the syntactic development of Portuguese children. Questions like: "Do children by a certain age manage to produce this syntactic structure?" led to several attempts in the construction of the items. Adaptation studies tests were taken from 288 children. The final standardization test with 40 items was taken by 852 children with normal language development with ages ranging from $3 ; 0$ to $6 ; 0$, in all city districts of Continental Portugal. The administration of the test took less than 30 minutes and the items were scored dichotomously.

To determine the tests internal consistency, the Lambda-2 coefficient was applied resulting in, $r=.874$, which is considered good. The test-retest reliability was also studied with 61 subjects from different age groups two months after the first testing. The result was satisfactory: $r s=.80, p<.001$. Construct validity was also investigated by correlating the scoring of the STSD-PT with another language test in 60 children. The correlation coefficient pointed to a significant relation between both tests ( $r s=.80, p<.001$ ). Age differentiation is also an evidence of score validity. The Pearson's coefficient value showed that there is a positive and significant relation between the children's age and their raw scores $(r=.66, p<$ .001). The psychometric measures seem to show that the STSD-PT is valid and reliable for use in clinical situations.

\section{References:}

Schlichting, L., Van Eldik, M. C., Lutje Spelberg, H. C., Van der Meulen, S., \& Van der Meulen, B. F. (1995). Schlichting Test for Language Production: Lisse: Swets Test Services. 


\title{
Section 2 Language in Community and Media
}

\author{
Julia Döring \\ Moderne "Peinlichkeit" oder peinliche Zeiten? Zum Verhältnis von Wort, Begriff und \\ Phänomen am Beispiel von „Peinlichkeit“
}

Googlet man den Ausdruck „peinlich“, findet man weit über 2.000.000 Ergebnisse. Angesichts der Tatsache, dass dieses Wort einen grundlegenden sozialpsychologischen Wirkungsmechanismus bezeichnet bzw. zumindest diskursiv als ästhetisches Urteil auf diesen referiert, ist es allerdings verwunderlich, dass noch im siebten Band von Grimms Wörterbuch von 1895 diese heute vorherrschende Bedeutung von „peinlich“/,Peinlichkeit“ gar nicht verzeichnet war. "Peinlichkeit“ in diesem Sinne scheint also, zumindest vom linguistischen Standpunkt aus gesehen, ein speziell neudeutsches Phänomen zu sein (vgl. Stroh 2005), eine Tatsache, die Fragen nach dem Verhältnis von Sprache und Wirklichkeit aufwirft: Ist uns entgegen aller medialer Peinlichkeitsinszenierungen in den vergangenen Jahrzehnten einfach mehr peinlich geworden? Lässt sich eine Entwicklungslinie in der entsprechenden Emotionskonstruktion nachzeichnen, die „von der archaischen Scham des ,Primitiven' über die verletzte Ehre des Menschen in ständischen Sozialverbänden bis hin zur [peinlichen] Blamage des modernen Subjekts geführt hat" (Neckel 1987: 80), sodass es anachronistisch wäre, von „Peinlichkeit“ in Mittelalter und Früher Neuzeit zu sprechen? Welche Rückschlüsse erlaubt dann der Bedarf eines speziellen Ausdruckes für jene Peinlichkeit, die das Verhalten anderer in uns auslöst, die „Fremdscham“ (seit 2009 im Duden)? Der Vortrag möchte diese und ähnliche Fragen aus kommunikations- und sprachwissenschaftlicher Perspektive erörtern, wobei am Beispiel von „Peinlichkeit" das allgemeine Verhältnis von Wörtern, Begriffen und Wirklichkeit diskutiert werden soll, welches bei Emotionsphänomenen besonders komplex zu sein scheint. Das Bezeichnete kann hier zwar abstrakt und unabhängig von der Bezeichnung als Begriffskonzept erfasst werden, doch im empirischen Phänomenbereich verschwimmt diese Grenze: Betrachtet man Emotionen nicht als Reaktionen auf objektive Reize, sondern als höchst kommunikativ konstruierte Phänomene, können sie in Gesellschaften mit entsprechendem Emotionslabel ja nicht nur eine andere soziale Bedeutung, sondern auch eine andere Erlebnisqualität besitzen als in jenen Gesellschaften, die nicht über ein solches Etikett verfügen (So fragt sich z.B. Miller: "What is the connection between the richness or poverty of a vocabulary of emotions and the richness or poverty of emotional experience? [...] Does it make a difference in the actual substance of the feeling to have a single word describe a particular emotional state rather than a variety of more paraphrastic or circumlocutional descriptions?" (Miller 1993: 99 f). Im Gegensatz zu den meisten anderen Emotionsvokabeln gibt das Peinlichkeitslabel darüber hinaus sowohl konkrete emotionale Betroffenheit als auch gleichzeitig schon das entsprechende Emotionskonzept wieder, Gefühlszustand und bewusste Gefühlsklassifikation vereinen sich in einem einzigen sprachlichen Ausdruck: „Peinlich!“

\section{Literatur:}

Miller, Ian William (1993): Humiliation. And Other Essays on Honor, Social Discomfort, and Violence. London: Cornell University Press. 
Neckel, Sighard (1991): Status und Scham: zur symbolischen Reproduktion sozialer Ungleichheit. Frankfurt am Main: Campus.

Stroh, Wilfried (2005): Ein Hoch auf die Peinlichkeit! In: Roediger, Thomas (Hrsg.) (2005): Peinlich, peinlich. Anthologie. Kurzgeschichten Wettbewerb 2005. Herbert Utz Verlag.

\section{Beatrix Fehse \\ Metaphern in Text-Bild-Gefügen. Verbale Metaphorik. Visuelle Metaphorik. Zeitmetaphern in der Anzeigenwerbung und der Gegenwartskunst}

Auf dem Junior Research Meeting möchte ich gern zeigen, wie ich Zeitmetaphern in Kunstwerken analysiere und deute, denn genau hierauf zielt meine Dissertation (s.o.) hin. Das heißt, ich könnte mir vorstellen, an einer kleinen repräsentativen Auswahl an Kunstwerken aus meinem Korpus meinen Analyse- und Deutungsweg vor- und zur Diskussion zu stellen. Etwa zwei Kunstwerke von zehn Künstlern und Künstlerinnen (zum Beispiel von Werner Büttner, Felix Droese, Jochen Gerz, Georg Herold, Thomas Huber, Anselm Kiefer, Christiane Möbus, Neo Rauch, Gabriele Schmidt-Heins und Thomas Schütte) könnte ich einspielen. Teils würde ich ihre Zeitmetaphorik erklären, teils würden wir sie durch die gemeinsame Betrachtung und Lektüre neu entdecken. Das von mir sehr erwünschte Gespräch böte zudem jedem Teilnehmer und jeder Teilnehmerin die Chance, die eigene Analyse und Deutung auszudrücken sowie fremde Analysen und Deutungen auf- und anzunehmen.

Die Werke meiner engeren Auswahl sowie meines gesamten Korpus' wurden vorwiegend in den 1970er bis 1990er Jahren angefertigt. Es sind Werke ganz unterschiedlicher Dimension: kleinere Skizzenblätter, größere durchkomponierte Gemälde, Objekte wie auch Objektkästen und Installationen im Museum sowie im öffentlichen Raum. Sie sind allerdings alle statisch und erfüllen zudem die zwei Kriterien, erstens aus Bild- und deutschsprachigen Textelementen zu bestehen und zweitens einen zeitlichen Aspekt zentral zu thematisieren.

Meine Unterstellung, auch Objekte seien Bilder, ist zwar eher unüblich, dürfte aber nicht wirklich überraschen. So wie man alles, was sich vor unseren Augen präsentiert und abspielt, als ein Wahrnehmungsbild bezeichnen kann, kann man auch alles Sichtbare, was zu einem Kunstwerk hinzugehört, als dessen Bild bezeichnen. Mit dem Sprung von der Zweidimensionalität in die Dreidimensionalität weitet sich lediglich die Sehfläche (Schmitz 2009) zu einem Sehraum aus. Auch in ihm können Texte und Bilder gemeinsame Bedeutungseinheiten bilden („Sehflächen sind Flächen, auf denen Texte und Bilder in geplantem Layout gemeinsame Bedeutungseinheiten bilden." Ulrich Schmitz: Sehflächenforschung. Eine Einführung, 2009: S. 3 unter: <http://www.linse.unidue.de/linse/publikationen/sehflaechenforschung.php>).

Entscheidend ist für mich, dass aus dem Zusammenspiel der Bilder und Texte dieser Sehflächen oder -räume Metaphern unterschiedlichster Strukturen erwachsen. Durch meine Vorauswahl beziehen sich diese alle auf das schwer ergründliche Phänomen der Zeit. Newton (1643 - 1727) fasst die Zeit quantitativ, Augustinus (354 - 430) qualitativ. Der absoluten Zeit, die nur die Vergangenheit und die Zukunft abmessen kann, steht scheinbar unversöhnlich die erlebte Zeit gegenüber, aus deren Gegenwart wir nicht herauskommen. Auf je eigene Art und Weise bergen die Kunstwerke in ihren Metaphern das eine oder das andere Merkmal der Zeit, aber nicht selten auch beide und regen so zur Reflexion an. 
Mich interessiert daran zum einen die Struktur dieser Metaphern, das heißt, ich verfolge die Leit-frage, wie die Metaphern auf Text und Bild verteilt sind. Zum anderen interessieren mich aber auch die Inhalte der Metaphern, das heißt, ich verfolge die Leitfrage, welche Metaphern durch die Zeitreflexion entstehen. Beide Leitfragen kann ich mit Rückgriff auf die Metapherntheorien von Weinrich (1958 - 1976) und Lakoff \& Johnson (1980/85) beantworten. Die Ausweitung der Metapher auf den visuellen Bereich stützen zudem mehrere Theorien zur visuellen Metaphorik seit Aldrich (1968/71). Sie ebnen den Weg für die Erweiterung der weinrichschen Metaphorik auf die bildliche Ebene.

\section{Antonia Unger, Katja Nikitin, Dipl.-Soz.wiss.}

Organisationskommunikation im mehrsprachigen Vergleich die Krisenkommunikation von BP während der Ölkatastrophe im Golf von Mexiko

Die Explosion der Ölplattform „Deepwater Horizon“ im April 2010 löste eine Umweltkatastrophe ohnegleichen aus. Um die Weltöffentlichkeit zu informieren und das Image des Konzerns British Petroleum (BP) zu retten, initiierte die PR-Abteilung des Unternehmens eine intensive mehrsprachige Online-Krisenkommunikation. In der Fachliteratur zur Krisenkommunikation wird wiederholt gefordert, dass Krisenmeldungen im Sinne einer One Voice Policy (Bruhn 2006) „mit einer Stimme“ kommuniziert werden sollen. Die sprachliche Realisierung einer One Voice Policy erweist sich bereits in einem monolingualen Kontext als komplex. Diejenigen, die mit der mehrsprachigen Kommunikation in international tätigen Unternehmen betraut sind, stellt sie jedoch vor besonders komplexe kommunikative Aufgaben.

Hier setzt der vorliegende Vortrag an, der im Rahmen eines Praxisprojekts zur mehrsprachigen Krisenkommunikation im Fahrbereich der Interkulturellen Wirtschaftskommunikation an der Universität Jena entstanden ist. Ziel war es, am Beispiel der originalsprachig englischen BP-Pressemitteilungen und deren deutschen Übersetzungen zu untersuchen, ob sich Muster sprachlicher Abweichungen feststellen lassen, die auf kulturell unterschiedliche Textkonventionen hindeuten. Zu diesem Zweck wurden die Originale und Übersetzungen mit dem systemisch-funktionalen Übersetzungsbewertungsmodell von House (2001) untersucht. Die Übersetzungsanalyse betrachtet die Verwendung von Stilmitteln, die im Metadiskurs (Hyland 2005) als bedeutsam angesehen werden. Damit werden Textmittel untersucht, die den Diskurs strukturieren, den Leser ansprechen und die Haltung des Autors markieren. Der Fokus der Projektanalyse liegt auf linguistischen Mitteln und Konstruktionen wie Aktiv-/Passivkonstruktionen, Modalität (Diewald 1999) und Konnektivität (Bührig \& House 2007).

Die systemisch-funktionale Übersetzungsanalyse zeigt, dass zwischen den englischsprachigen Originalen und den deutschen Übersetzungen signifikante Abweichungen hinsichtlich der Bewertung der Krise und der Rolle BPs existieren: In den englischsprachigen Originalmeldungen wurden die Maßnahmen BPs durch den Gebrauch von Aktivkonstruktionen und "material verbs" (Halliday \& Matthiesen 2003) sowie der Darstellung BPs als Agens aktiver kommuniziert. Abschließend wird die Anwendbarkeit der Ergebnisse zur Optimierung mehrsprachiger Krisenkommunikation erörtert.

\section{Literaturangaben}


Bruhn, Manfred (2006): Integrierte Unternehmens- und Markenkommunikation. SchäfferPoeschel.

Bührig, Kristin \& Juliane House (2007): Linking Constructions in Discourse across Languages: In: Rehbein, Jochen et al eds. Connectivity in Grammar and Discourse. Amsterdam: Benjamins, 345- 366.

Diewald, Gabriele (1999): Die Modalverben im Deutschen: Grammatikalisierung und Polyfunktionalität. Tübingen: Niemeyer (Reihe Germanistische Linguistik 208).

Halliday, Michael A.K. \& Christian Matthiessen (2003): An Introduction to Functional Grammar (3. Auflage) London: Arnold.

House, Juliane (2006): How do we know when a translation is good? In: Steiner, Erich and Colin Yallop (eds.): Exploring Translation and Multilingual Text Production: Beyond Content. Berlin: Mouton de Gruyter, $127-160$.

Hyland, Ken (2005): Metadiscourse: Exploring interaction in writing. London \& New York: Continuum

\section{Julia Steube, M.A.}

Laienlexikografie im Internet

Das Internet bietet heute eine unüberschaubare Masse an Wörterbüchern und Lexika verschiedenster Qualität und Herkunft. Diese Vielfalt steht jedoch in einem Missverhältnis zu den bevorzugten Untersuchungsgegenständen und Forschungsinteressen der aktuellen Wörterbuchforschung. Sie beschäftigt sich im Rahmen der „kritischen Wörterbuchforschung" fast ausschließlich mit Werken, die in Verlagen oder akademischen Wörterbuchwerkstätten, also unter Mitarbeit professioneller Lexikografen oder Redakteure entstehen. Die breite Masse an nichtprofessionell erstellen Nachschlagewerken, die im Internet anzutreffen ist, wird in der Wörterbuchforschung weitestgehend ignoriert oder im Rahmen von Untersuchungen zu Online-Wörterbüchern undifferenziert unter diesen „neuen“ Nachschlagewerkstypus subsummiert. Selbst die Wikipedia, als meist genutztes Online-Nachschlagewerk der Welt, wird im Rahmen der Wörterbuchforschung verhältnismäßig stiefmütterlich behandelt. So lässt sich z.B. in der „Online-Bibliografie zur elektronischen Lexikografie" des Instituts für Deutsche Sprache (OBELEX, http://hypermedia.ids-mannheim.de/pls/lexpublic/bib.ansicht (Stand: 06.01.2011) nicht ein einziger Titel finden, der sich explizit der Online-Enzyklopädie widmet. Folgt man WIEGAND (1998: 39) wie auch dem naheliegenden Verständnis von "Wörterbuchforschung", so gehören aber selbstverständlich auch solche Nachschlagewerke zu ihren Untersuchungsgegenständen, die in „nichtwissenschaftlichen“ lexikografischen Prozessen entstanden sind (Wiegand (1998: 39) bezieht sich auf den Bereich der Sprachlexikografie, analog kann dies aber für die Sachlexikografie gelten).

Dass die Kriterienkataloge der kritischen Wörterbuchforschung nicht in der gewohnten Form auf nichtprofessionell erstellte, also laienlexikografische Nachschlagewerke angewandt werden können, leuchtet ein - jegliche Verbesserungsvorschlage würde die Macher der Nachschlagewerke aufgrund des fehlenden Kontakts zum metalexikografischen Fachdiskurs nicht erreichen. Welche Aspekte laienlexikografischer Nachschlagewerke für die Wörterbuchforschung dennoch von Interesse sein können bzw. sollten, erste Ergebnisse und deren Nutzen für die Metalexikogafie - diese Punkte sollen im Rahmen des Vortrags geklärt werden. Der Fokus liegt dabei auf sachbezogenen Online-Nachschlagewerken, die jenseits der Wikipedia von lexikografischen Laien erstellt wurden. 


\section{Literatur:}

Wiegand, Herbert Ernst (1998): Wörterbuchforschung. Untersuchungen zur Wörterbuchbenutzung, zur Theorie, Geschichte, Kritik und Automatisierung der Lexikographie. Berlin [u.a.]: de Gruyter.

\section{Tanja Kaya, B.A.}

Bilingual Pragmatic Competence: The Use of the Speech Act of Apology by Turkish-German Bilingual Students

In this presentation, an ongoing empirical study regarding the pragmatic competence of Turkish-German bilinguals will be summarized. The study aims at measuring the use of the speech act of apology. The main research questions that prompted this study are; "What apologizing strategies do Turkish-German bilinguals use? What kind of strategies do TurkishGerman bilinguals implement linguistically? What kind of politeness strategies do they apply both on the linguistic and the pragmatic level? Do they make bi-directional transfer? How does the transfer affect the strategy usage?"

The study is explorative and descriptive in nature using a laboratory method. The sample of the study consists of 70 Turkish-German bilingual university students. For data collection, a set of discourse role-play tasks (DRPT) for both languages have been developed. Subsequently, the subjects were assigned to play the situations first in Turkish and after a two-month break in German so as to reduce the influence of the language usage. These role plays had been video-taped, which constitutes the primary source of the data. Furthermore, a questionnaire had been designed to collect additional data on demographic information, subjects' language acquisition, language learning process and their parents' educational background. Discovering characteristics of bilinguals' language usage namely the pragmatic competence is of great importance for SLA and SLT research. Therefore; it is expected that the key findings of the study will serve for the development of innovative and qualitative language programs.

\section{Juliane Mroz, M.A.}

Verständlichkeit von Anglizismen und Kurzwörtern in Internetfachsprache und Internetslang

Auch wenn die Zahl der Internetnutzer in Deutschland jährlich steigt (siehe Nonliner-Atlas unter www.initiatived21.de), gibt es bestimmte Bevölkerungsgruppen, bei denen die Nutzerzahlen stagnieren - allen voran Frauen im Seniorenalter mit niedriger formaler Bildung. Die Ergebnisse einer qualitativen Untersuchung im Rahmen meiner Magisterarbeit deuten darauf hin, dass Internet-Einsteiger im Alter von 60 Jahren an sich häufig durch die internetspezifische Fach- und Umgangssprache überfordert fühlen. Dennoch hat die Untersuchung auch Hinweise darauf geliefert, dass die terminologische Kompetenz der Probanden wesentlich höher ist als von ihnen selbst angenommen. Internetspezifische Anglizismen und Kurzwörter konnten die Befragten korrekt erklären und anwenden, auch wenn ihnen die Übersetzung beziehungsweise Langform nicht bekannt war.

Wie gut auf englischen Lexemen basierende internetspezifische Termini von Nutzern verschiedener Altersgruppen und mit unterschiedlichem Erfahrungsstand verstanden 
werden, soll eine quantitative Untersuchung zeigen, die Teil meiner Dissertation ist. Welche Termini untersucht werden, ermittle ich vorher durch eine Korpusuntersuchung interessant sind sowohl sehr häufig als auch sehr selten vorkommende Wörter sowie eine Vergleichsgruppe von Wörtern mit mittlerer Frequenz.

Die Nutzerbefragung soll zeigen, inwiefern Anglizismen auch dann verstanden werden, wenn die deutsche Übersetzung nicht bekannt ist (oder es keine gibt), und ob die Befragten die Bedeutung internetspezifischer Kurzwörter auch dann korrekt wiedergeben können, wenn sie die Langform nicht kennen. Die Auswertung der Befragung soll Erkenntnisse darüber bringen, welche morphologischen Eigenschaften einen Einfluss auf die Verständlichkeit der Wörter haben (beispielsweise der Grad ihrer Integration in die deutsche Sprache).

Um mehr über die Hintergründe des Verstehens oder Nichtverstehens zu erfahren, werde ich einige Probanden zusätzlich interviewen und die Interviews qualitativ auswerten. Dabei will ich auch ergründen, inwiefern Probleme mit internetspezifischen Termini das Surfverhalten der Nutzer beeinflussen.

\section{Katharina König}

Indirekte Formulierungen in narrativen Interviews

Narrative Interviews haben sich als Methode der qualitativen Sozialforschung längst etabliert. Auch in linguistischen Untersuchungen werden sie verschiedentlich eingesetzt: $\mathrm{Ob}$ als qualitative Interviews zur Elizitierung von biographischen Hintergrundinformationen oder als Sprachbiographien zur Reflektion von individuellem Erwerb und Nutzung von Mehrsprachigkeit in monolingual geprägten gesellschaftlichen Zusammenhängen (vgl. Franceschini 2002). Haben sich viele Analysen im Bereich der Sprachbiographien bislang mit der Untersuchung von zentralen Topoi des Spracherwerbs (Ohm 2008) oder der narrativen Ausgestaltung (Fünfschilling 1998) befasst, so soll in diesem Beitrag in einer gesprächsanalytischen Ausrichtung nach wiederkehrenden Formulierungsverfahren im Rahmen der kommunikativen Gattung (Günthner 1995) "Narratives Interview" gefragt werden. Anhand eines Korpus von 15 narrativen Interviews mit mehrsprachig aufgewachsenen vietnamesisch-stämmigen Frauen und Männern in Deutschland wird der sequentielle Aufbau und die Funktion von indirekten Formulierungen betrachtet: Thematisiert werden Kategorische Formulierungen (Ayaß 1999) der Form "wenn man... dann..." und Indefinitformulierungen mit dem Indefinitpronomen "man". Es soll gezeigt werden, dass diese Formulierungsstrategien positionierungsrelevant sind (LuciusHoene/Deppermann 2004) und zur Konstruktion eines moralischen Rahmens der Gattung "Narratives Interview" beitragen.

\section{Literatur:}

Ayaß, Ruth (1999): Form und Funktion kategorischer Formulierungen. In: Bergmann, Jörg/ Thomas Luckmann (Hrsg.): Kommunikative Konstruktion von Moral. Band 1: Struktur und Dynamik der Formen moralischer Kommunikation. Opladen: Westdeutscher Verlag, 106-124.

Franceschini, Rita (2002): Sprachbiographien: Erzählungen über Mehrsprachigkeit und deren Erkenntnisinteresse für die Spracherwerbsforschung und die Neurobiologie der Mehrsprachigkeit. In: Bulletin VALS-ASLA 76, 19-33.

Fünfschilling, Johanna (1998): Spracherwerb als Teil der Biographie: Zur Versprachlichung 
von Erwerbserinnerungen in narrativen Interviews. In: Mondada, Lorenza/Georges Lüdi (Hrsg.): Dialogue entre linguistes. Recherches en linguistique à I'Institut des Langues et Littératuers Romanes de l'Université de Bâle. 65-79.

Günthner, Susanne (1995): Gattungen in der sozialen Praxis. Die Analyse "kommunikativer

Gattungen" als Textsorten mündlicher Kommunikation. In: Deutsche Sprache 3, 193-

218.

Lucius-Hoene, Gabriele/Arnulf Deppermann (2004): Narrative Identität und Positionierung. In: Gesprächsforschung - Online-Zeitschrift zur verbalen Interaktion 5, 166-183.

Ohm, Udo (2008): Zweitsprachenerwerb als Erfahrung. Eine qualitativ-explorative Untersuchung auf der Basis narrativer Interviews. Habilitationsschrift an der Friedrich-Schiller-Universität Jena.

\section{Eva Zumhasch}

The Use of English in Hong Kong Rap: An Example from 24Herbs

English in Hong Kong is a legacy of British trade and colonialism and until today has coofficial status in the now Special Administrative Region (SAR) of the People's Republic of China. Its status as a second language, however, is still debated. Yet, some features characteristic of the English variety spoken in Hong Kong have been identified, such as the simplification of word-final consonant clusters, the confusion of $/ \mathrm{r} /$ and $/ \mathrm{I} /$ in certain environments, or the absence of weak forms in unstressed syllables (cf. Bolton 2005).

In its colonial past, Hong Kong's normative orientation was mainly towards the British English standard, due to globalization and the spread of the new media; however, other forms of English have reached the SAR. One of the dominant varieties of globalization seems to be American English prevalent in contexts such as the internet or popular culture. Popular music, for instance, finds great acceptance among the younger generation in Hong Kong and there is quite a few musicians who produce songs in English.

The presentation will explore which forms and varieties of English occur in a selected rap song from a local hip hop group. The genre of rap has predefined conventions and norms as well as features of language use that can be considered a global phenomenon. Global rap language includes, for instance, the absence of post-vocalic $/ r /$, copula deletion, formulaic expressions and phrases, or slang words (cf. Alim 2006). Apart from the rap language, the presentation attempts to show other features of language use, such as those that Trudgill (1997) has defined as stereotypical American English features - e.g. the voicing of intervocalic /t/, the presence of prevocalic / $r$ / in words such as girl, or the use of an unrounded / $\mathrm{a} /$ instead of $/ \mathrm{b} /$ in words such as body - next to those that can be considered Hong Kong English features outlined earlier.

The Hong Kong hip-hop group 24Herbs comprises six members of different backgrounds: some are local Hong Kong Chinese while others are overseas returnees. They rap in two languages, English and Cantonese. The locals usually rap in Cantonese while the returnees rap in English. The hybrid title of 24Herbs' song "Respect Tou Pok Kai" is a classic example of 'flipping the script' in hip hop, i.e. turning the negative connotations of the (in this case Cantonese) swear words into positive ones. Other features of rap language can also be identified in the song, such as the absence of post-vocalic $/ r /$ at the end of words as in never quitter, or the use of formulaic phrases such as keep it real. The rappers of $24 \mathrm{Herbs}$ also use American English features such as the voicing of intervocalic $/ t /$, or $/ a /$ instead of $/ \mathrm{p} /$, as in 
everybody. Hong Kong English features present in the song include word-final consonant cluster simplification as in respect, hybrid words, or the use of locally coined vocabulary.

\section{References}

Alim, H. Samy (2006). "Hip Hop Nation Language." In: Edward Finegan, Edward, John R.

Rickford (eds.): Language in the USA. Themes for the Twenty-first Century. Cambridge: Cambridge UP. 387-409.

Bolton, Kingsley (2005) (ed.) Hong Kong English. Autonomy and Creativity. Hong Kong: Hong Kong UP.

Trudgill, Peter (1997). "Acts of Conflicting Identity: The Sociolinguistics of British Pop-song Pronunciation." In: Nikolas Coupland, Adam Jaworski (eds.). Sociolinguistics. A Reader and Coursebook. Modern Linguistics Series. Baskinstoke: Macmillan Press. 251-265.

\section{Section III Language in Education}

\section{Stefanie Couve de Murville}

Wie erfolgreich ist bilingualer Sachfachunterricht wirklich? -Lernstandserhebungen in einer immersiven Grundschule

Bilinguale Konzepte erfreuen sich besonders in Grundschulen immer größerer Beliebtheit. Doch wie erfolgreich ist der bilinguale Sachfachunterricht wirklich? Im Mittelpunkt der wissenschaftlichen Begleitung einer norddeutschen Grundschule steht die Dokumentation verschiedener Leistungsstände innerhalb zweier immersiv unterrichteter Klassen, welche mit Ausnahme des Unterrichtsfaches Deutsch - ausschließlich auf Englisch unterrichtet werden. Die Untersuchung der Kompetenzprofile der Klassen verfolgt neben der fremdsprachlichen Entwicklung auch die Untersuchung der Leistungen in den Sachfächern. Durch den Einsatz ausgewählter Schulleistungstests in den Fächern Deutsch und Mathematik sowie anhand von Unterrichtsaufzeichnungen aus dem Sachunterricht werden die Lernstände der immersiv unterrichteter Klassen mit denen der nicht immersiv unterrichteten Parallelklassen verglichen. Neben der Untersuchung der Leistungen in den Sachfächern wird auch eine qualitative Analyse von Unterrichtssequenzen unterschiedlicher handlungs- und produktionsorientierter Sachunterrichtsthemen verfolgt. Ein besonderes Augenmerk soll dabei auf sprachliche und parasprachliche Kommunikationsstrategien, u.a. negotiation of meaning, in der Lehrer-Schüler-Interaktion gelegt werden.

\section{Emilie Magnat}

A multisensory approach of learning English L2 to work in tomorrow world

The following proposition of presentation examines the question of a multisensory approach to second language learning and more precisely English L2 in France. Even more in the future, people will move and work with other people from other countries and other languages and so, they will need a common language. In this respect, I support Jenkins' idea of "intelligibility" of English as an international language (Jenkins, 2000) and suggest that we should consider this target from the early teaching at school. If we look closer at Jenkins' 
empirical research, we notice that the pronunciation emerged as the main problem for understanding between speakers of different L1 (Jenkins, 2000: 19). Beside, various studies in Second Language Acquisition show that speakers of $L 2$ tend to be disturbed by transfers coming from their L1 (see for example Jenkins, 2000: 88; for the impact of French on the learning of English see Kanta \& Rey, 2003). Hence, we need to guide learners in the development of a phonological awareness in L2. This basis will then be determinant in their reading and writing performance (Adams, 1990).

Studies in cognitive psychology present the positive impact of a multisensory approach to develop phonological awareness in first language acquisition (Bara, Gentaz, \& Colé, 2007).

Researchers introduced haptic exploration of letters before testing learners' reading performance. In such a connectionist approach (see "Parallel Distributed Processing" by Rumelhart \& McClelland, 1987), gestures make the connection between the orthographic information and its phonological counterpart. We hypothesize that this approach can be used to develop phonological awareness in the learning of English L2 for French young learners. At a time when new technologies such as Interactive Whiteboards are set up in classroom, we should investigate whether the technical potential of the tool (haptic function) can be efficient for a cognitive development. At the light of the results, the PhD research can lead to the development pedagogical materials. 


\section{Dr. Marta Janachowska-Budych}

Literatur und das kulturelle Gedächtnis - Forschungsbericht und
Didaktisierungsmöglichkeiten

Als gesamtkulturelles und interdisziplinäres Phänomen genießt das Thema ,Gedächtnis' schon ungefähr drei Jahrzehnte lang ein verstärktes Forschungsinteresse. Die facettenreiche Verbindung zwischen Gedächtnis und Kultur wird auch intensiv in der Literaturwissenschaft exploriert. Die Forschungslage zur Verflochtenheit zwischen Literatur und Gedächtnis ist umfassend und fokussiert vor allem zwei Konzepte - das des Gedächtnisses der Literatur sowie das des Gedächtnisses in der Literatur. Relativ wenig erforscht bleibt immer noch das dritte literaturwissenschaftliche Gedächtniskonzept, nämlich Literatur als Medium des kulturellen Gedächtnisses. Aus diesem Grund versteht sich das Referat als Versuch, folgende Fragen zu beantworten: 1. Was ist der Zusammenhang zwischen der Literatur und dem kulturellen Gedächtnis? 2. Welche Wirkung hat Literatur innerhalb der Erinnerungskultur? 3. Was unterscheidet Literatur von anderen Medien des kulturellen Gedächtnisses? 4. Woraus resultiert das erinnerungsmediale Potential der Literatur? Darüber hinaus, soll das literarische Potential der Gedächtnisbildung und Gedächtnisreflexion kurz am Beispiel der Werke von Elfriede Jelinek und Olga Tokarczuk geschildert werden.

Nach dem einleitenden Forschungsbericht über die Konvergenzpunkte zwischen der Literatur und dem kulturellen Gedächtnis sollen die Didaktisierungsmöglichkeiten des Konzeptes des kulturellen Gedächtnisses - vor allem in Bezug auf die Fremdsprachen- und Literaturdidaktik - vorgestellt werden. In diesem Teil des Referats sollen solche Aspekte wie das kulturelle Gedächtnis als ein Teil des interkulturellen Lernens, Didaktisierung des Konzeptes von Erinnerungsorten oder die Integrierung der Gedächtnisthematik in den Literaturunterricht in den Mittelpunkt der Erwägungen rücken.

\section{Joanna Kic-Drgas}

Zwischen Instruktion und Konstruktion- neue Perspektive für den Fremdsprachenunterricht im Seniorenalter

Angesichts der sozialen und demographischen Änderungen in der Welt gewinnt der von Viëtor (1979:9) geäußerte Satz („Der Sprachenunterricht muss umkehren“) an Aktualität. Die gegenwärtige gesellschaftliche, politische und wirtschaftliche Situation auf der Welt verursacht die Diskussionen um die neue Form des Fremdsprachenunterricht (vgl. Szczodrowski 2006, 2010, Chudak 2007, Grucza 2007, Żylińska 2007, Oebel 2010).

Die von den Wissenschaftlern dargestellten Konzepte entdecken die Interdisziplinarität und Komplexität des Fremdsprachenunterrichts, was im Kontext der Sprachpraxis nicht nur einmalige Änderung, sondern permanente Modellierung bedeutet. Beachtenswert ist jedoch neben der horizontalen Differenzierung der Methoden, Techniken und Ansätze, auch das Bewusstsein vertikaler Unterschiede zwischen bestimmten Lernergruppen.

Eine der neuen Lernergruppen, die wegen der mangelnden Forschung eine große Herausforderung an die moderne Glottodidaktik stellt, sind Senioren (verwendet man in diesem Buch die maskulinen Formen: Lehrer, Lerner, Senior, so sind männliche und weibliche Personen gleichmassen gemeint. (vgl. dazu. Duden 4 1995:196).

Die Heterogenität der Senioren wird in der vorliegenden Dissertation noch mehrmals betont, jedoch was die Gruppe einigt, ist die gemeinsame Lerntradition, die betrachtend den durchschnittlichen Alter von 60 Jahren in den glottodidaktischen Grundgedanken der 70er 
Jahre letzten Jahrhunderts verankert ist. Gezielt wird deswegen in dem folgenden Kapitel ein kritischer Blick auf die Methoden geworfen, die derzeit (und vielleicht immer noch) in der Fremdsprachendidaktik Anwendung fanden, um die Spezifik der Wiederlerner zu verstehen um die Unterrichtspraxis durch Einsatz angepasster Materialien seniorenorientierter gestalten zu können.

Gleichzeitig wird die Tatsache berücksichtigt, dass die Senioren im Laufe der 30-40 Jahren beruflicher Betätigung einerseits Erfahrungen gesammelt haben, andererseits auch sich an Selbständigkeit im Entscheidungstreffen gewöhnt haben. Dies beeinflusst die Betrachtensweise der Welt und die Präferenzen der Lerner im Alter. Anbetracht dessen wird mit den Grundsätzen der konstruktivistischen Lerntheorie konfrontiert um die Frage nach deren Anwendung in der Unterrichtspraxis zu beantworten.

Letztendlich wird anhand der Analyse beider Konzepte (Instruktion und Konstruktion) nach dem Einfluss auf die (Re-)Konstruktion des linguistischen Wissens bei Senioren gefragt und die Rahmen des geragogischen Fremdsprachenunterrichts werden geprägt.

\section{Dominik Rumlich}

Learners' performance in written English: Do CLIL students achieve higher levels of accuracy than their peers in 'regular' classes?

CLIL (immersion) teaching is deemed to be one of the most, if not the most promising approach in language education and has been a red-hot topic in academia ever since it emerged.

Apart from the fact that linguistic correctness is a contentious matter in language education per se, CLIL classes represent a particularly intriguing area of research: The primacy of message before accuracy, id est a focus on meaning, is supposed to be its governing precept. So what effect does this then exert on students' linguistic accuracy? To what extent does this affect other variables such as students' motivation and their attitude towards their English classes? As the latter is bound to wield positive influence on students' performance in foreign language teaching classes, the potential detrimental effects of the former might be mitigated. But even examining empirical research, one cannot answer the questions raised in a satisfactory way due to a substantial academic void in this area of German CLIL teaching. To tackle these issues, I have conducted a quasi-experimental study based on 45 secondary students and their performance in exams. This led to true-to-life language samples with a genuine focus on meaning. Furthermore, these students filled out an additional questionnaire which addressed potentially influential variables such as their motivation, attitudes and their exposure to English outside the language classroom. While multiple statistical analyses of the resulting dataset confirm the basic assumption that CLIL students' written English is indeed more accurate, they also bear some considerable surprises with respect to the other variables examined. 


\section{Verena Möller}

\section{English Passive Constructions in the Interlanguage of German Learners}

In recent years, the educational system in Baden-Württemberg, southwest Germany, has undergone several major changes. 'Immersive-reflective' English lessons have been introduced at the primary level. In secondary schools, more and more CLIL (content and language integrated learning) programmes are being established. But do students' interlanguages change along with the educational setting?

Considering the different types of input that learners may be exposed to, the use of the English passive is considered to be indicative of possible structural differences between the interlanguages of the various groups of learners. To study their use of passive structures, written learner language will be collected and compiled in an electronic corpus, whilst controlling learner variables like motivation and general as well as language-related cognitive capacities by means of psychological testing. A tokenizer, lemmatizer, POS-tagger and, possibly, a parser will be used to prepare the written learner data for automatic or semi-automatic analysis, such that the learner texts can be compared to a target norm. The norm that learners can be assumed to aspire to is to be defined by the written input received in the classroom, compiled and annotated in a teaching materials corpus. From the analysis of the two corpora, insights may be gained with respect to the part that different degrees of learning and acquisition play in the development of a learner's interlanguage. The information obtained may serve as an empirical basis to answer questions arising in connection with the teaching of English as a foreign language and in immersive programmes, as well as with curriculum design.

The paper will describe the role of the educational setting as the basis of the research hypothesis, the compilation of the target norm corpus as well as the procedures involved in the collection of learner language and metadata and the compilation of the learner corpus.

\section{Annelien De Geest \\ Entering university: a process of acculturation}

Research has established that there is a tension between the expectations of students and staff upon entering university. Even though acculturation and self-efficacy within an academic setting are an intensively studied domain (see among others Van de Poel \& Brunfaut, 2004)., only one study on language students has been carried out at the University of Antwerp (Darlaston-Jones et al, 2003; Kimmins \& Stagg, 2009; Latham \& green, 1997; Pajares, 1996; Bandura et al, 1992, etc.). Even then, acculturation was studied in retrospect, from the point of view of master's students. To compensate for this drawback, my own research will focus on freshmen in an attempt to identify the features of the 'culture shock' or acculturation while it is still going on. My main aim is to find out how students perceive themselves and their role within the academic community and how this correlates with their relative academic success or failure.

In order to gain insight into the process of acculturation, my (longitudinal) study consists of three questionnaires filled out by first year students of English at the University of Antwerp. In total, about 130 students took part. The first questionnaire was handed out at the start of the year in an academic writing class, the second one at the end of the semester in the same class and the third one in the second semester after the students had received their first 
exam results. The study will culminate in in-depth semi-structured interviews with a selection of the student population.

Based on previous research, initial wonder and familiarization with the new community is expected to evolve into some sort of shock. Presumably this shift will take place right before the exam period or after students receive their grades. This first moment of 'feedback' is expected to have a profound effect on how they see themselves throughout their entire academic career.

\section{Agnieszka Świrko}

Die blinden Schüler und der Fremdsprachenunterricht

Viele blinde und sehbehinderte Menschen leiden vor allem unter den Problemen im Umgang mit der sehenden Umwelt, unter deren Unverständnis und Unbedachtsamkeit. Wenige von Sehenden können sich vorstellen, plötzlich nichts mehr zu sehen. Alles ist dunkel oder, erscheint nur noch in grauen Schattierungen. Die Blinden sind in einigen Lebensbereichen eingeschränkt, z.B. im lebenspraktischen Bereich: beim Essen mit Messer und Gabel oder später im Erwachsenenalter beim Unterschreiben eines Dokumentes. Es gibt die unterschiedlichsten Gründe, warum es neben der Geburtsblindheit zu einer Erblindung kommen kann: zunehmendes Alter, Unfälle oder verschiedene Krankheiten können die Ursache für den Verlust der Sehfähigkeit sein. Der Umgang und das Miteinander zwischen Blinden beziehungsweise Sehbehinderten und gesunden Menschen sollte so "normal“ wie möglich sein. Für Blinde gibt es eine Vielzahl von Hilfsmitteln. Dazu gehören zum Beispiel speziell ausgestattete Lesegeräte, Computer, Farberkennungsgeräte, Hörbücher oder -filme, sprachgesteuerte Haushaltsgeräte wie Waage, Mikrowelle, Waschmaschine, Fieberthermometer, Zebrastreifen mit Tongeräuschen und vieles mehr, deren Kosten zum Teil von den Krankenkassen übernommen werden. Es gibt beispielsweise ein Farberkennungsgerät, so groß, wie ein Handy. Es hilft den Blinden zu erkennen, welche Farbe ein Pulli oder die Socken haben. Seit den 90er Jahren wurden auf der ganzen Welt Servicestellen eröffnet, die Menschen mit Behinderung speziell bei der Berufsorientierung und beim Fußfassen am allgemeinen Arbeitsmarkt unterstützen. Blinde oder sehbehinderte Menschen wollen in der Regel keine Sonderbehandlung, Mitleidsäußerungen oder aufgedrängte Hilfe. Die Frage, ob man behilflich sein kann, ist deshalb aber nie falsch. Der Betroffene wird die Hilfe gerne annehmen oder aber darauf hinweisen, dass er gut alleine zurechtkommt. Daraus ergeben sich folgende Fragen:

- Wie sieht heute der Zugang zu Bildung für Menschen mit Blindheit von Geburt an und im späteren Alter aus?

- Wie kann ich selbst helfen?

- Wie soll der Fremdsprachenunterricht aussehen, damit die Blinden eine Fremdsprache richtig lehren?

Das Thema und die Aufgabenstellung des Beitrages stellen sich als ein Stück Neuland dar.

Der Beitrag soll uns beibringen, dass die Blindheit bei der Fremdsprachenaneignung auf keinen Fall stört und die Blinden, so wie die Sehenden berufstätig sein können. Dies möchte ich unter anderem diskutieren. 


\section{Section IV Language in Professional Settings}

\section{Petra Klimant \\ Mentale Erschöpfung und Übersetzungsqualität}

Seit über zwanzig Jahren hat sich die Übersetzungsprozessforschung als noch recht junge Disziplin der Translationswissenschaften unter anderem der stetigen Optimierung von empirischen Methoden und Instrumenten zur Datenerhebung verschrieben, deren Ergebnisse darüber Aufschluss geben sollen, „was in den Köpfen von Übersetzern vorgeht" (Krings 1986). Zwar wurde dem magischen Dreieck des Projektmanagements mit seinen drei Eckpfeilern Zeit, Qualität und (mentale) Ressourcen bereits auch im übersetzungsprozessualen Kontext Beachtung geschenkt (Bayer-Hohenwarter 2008), doch bleiben einige Fragen, etwa zum potentiellen Kausalzusammenhang zwischen längeren Übersetzungszeitspannen und der Übersetzungsqualität immer noch unbeantwortet.

Auf der Suche nach Antworten auf einige dieser Fragen bildet eine empirisch-experimentelle Studie den Kern des hier vorgestellten Forschungsprojekts, bei der die Auswirkungen mentaler Erschöpfung und damit einhergehender, sinkender Aufmerksamkeit auf die Leistungsfähigkeit des Übersetzers und somit auf die Übersetzungsqualität untersucht werden. Nichtprofessionelle und professionelle Versuchspersonen übersetzen in dem Schreiblogging-Programm Translog einen längeren Text (Sprachenpaar Spanisch/Deutsch) und füllen sowohl vor als auch nach dem Übersetzungsexperiment jeweils einen studienrelevanten Fragebogen aus. Eine Reihe von Gutachtern bewertet im Anschluss an das Übersetzungsexperiment die Qualität der Übersetzungen in ihrer Gesamtheit. Außerdem werden in einer weiteren Bewertungsphase sprachliche Merkmale, die die Übersetzungsqualität beeinflussen, hervorgehoben. Die verschiedenen qualitativen und quantitativen Prozess- und Produktdaten werden schließlich trianguliert, sprich, zur Optimierung der Datenvalidität miteinander kombiniert.

Der Schwerpunkt des Vortrags liegt auf den empirischen Datenerhebungsmethoden und instrumenten, deren Vor- und Nachteile im Hinblick auf das Vorhaben umrissen werden.

Krings, H. P. (1986). Was in den Köpfen von Übersetzern vorgeht. Eine empirische Untersuchung zur Struktur des Übersetzungsprozesses an fortgeschrittenen Französischlernern. Tübingen: Narr.

Bayer-Hohenwarter, G. (2008). Alles nur eine Frage der Zeit? - Methodologische Überlegungen zu Zeit(druck) und Translation. The Journal of Specialised Translation, 9, 108-131.

\section{Andrea Hunziker Heeb}

A window onto the translator's mind: how process research helps shed light on problem awareness

Producing a text involves a demanding cognitive process. Issues like cohesion, level of language and terminology have to be considered. Therefore, being aware of such requirements and the problems they could pose is important. In order to investigate how problem awareness works, it makes sense to track and describe it where it is actually applied: not in the final product but in the process of text production. 
As regards text production in the translation process, this has been done in a sub-study of a nationally funded research project, 'Capturing translation processes'. So as to investigate problem awareness, MA students in translation and experienced professionals were monitored while they were translating a short text, using methods such as screen recording, eye tracking and retrospective verbal protocols. The analysis was based on a specific translation problem they encountered in translating the source text from their second language (English) into their first language (German). Preliminary results suggest differences in problem awareness between the two groups. That is, both groups recognised the selected problem but the professionals seemed to categorise it differently and, consequently, follow other paths to find a solution. This difference in behaviour may be explainable by models of translation competence acquisition, and its description may therefore allow the release of expert knowledge.

In addition to translation, process research has provided insights into text production in domains such as journalism, children's writing and academic writing. No matter what kind of problem in text production is concerned, the use of process research techniques can open a window onto the writer's mind.

\section{Selected references:}

Göpferich, S. (2008) Translationsprozessforschung. Stand, Methoden, Perspektive. Translationswissenschaft 4. Tübingen, Narr.

Göpferich, S., Jakobsen, A. L. and Mees, I. M. (eds) (2008) Looking at eyes. Eye-tracking studies of reading and translation processing. Copenhagen Studies in Language 36. Copenhagen, Samfundslitteratur.

Göpferich, S. and Jääskeläinen, R. (2009) Process research into the development of translation competence: where are we, and where do we need to go? Across Languages and Cultures, 10(2), pp. 169-191.

Krings, H. P. (1986) Was in den Köpfen von Übersetzern vorgeht. Eine empirische Untersuchung zur Struktur des Übersetzungsprozesses an fortgeschrittenen Französischlernern. Tübingen, Narr.

Muñoz Martín, R. (2010) On paradigms and cognitive translatology. In: Shreve, G. M. and Angelone, E. (eds) Translation and Cognition. Amsterdam, John Benjamins, p. 169-187.

PACTE (2000) Acquiring translation competence: hypotheses and methodological problems in a research project. In: Beeby, A, Ensinger, D. and Presas, M. (eds) Investigating Translation. Amsterdam, John Benjamins, pp. 99-106.

\section{Gabriela Gorąca}

Linguisten und ihre Kompetenzen in linguistischen Berufen

Linguisten sind in Medienberufen stark gefragt. Sind die Studierenden der angewandten Linguistik ausreichend auf den Beruf des Journalisten oder Copywriters vorbereitet? Welche Kompetenzen werden bei den Studentinnen und Studenten im Studium der linguistischen Fachrichtung entwickelt? Entsprechen ihr Wissen und Können den Anforderungen, die im Medienfeld an die Linguisten gestellt werden? Im vorliegenden Beitrag wird aufgezeigt, wann der Absolvent der angewandten Linguistik als kompetent für die Arbeit in Medien bezeichnet werden kann. 


\section{Julia Spanke}

Verständigungsprobleme. Eine empirische Analyse am Beispiel der industriellen Prozessmodellierung

Jemanden nicht verstanden zu haben oder selbst nicht verstanden worden zu sein, ist ein alltägliches Phänomen. Verständigungsprobleme sind so alltäglich, dass sie uns manchmal nur in Situationen bewusst werden, wo wir ihrer Bearbeitung große Aufmerksamkeit schenken müssen (Fiehler 2002, Kindt/Weingarten 1984, Selting 1987). Der Beitrag fokussiert Verständigungsprobleme in einem speziellen Bereich der Unternehmenskommunikation, nämlich in der Anwendung industrieller Prozessmodellierungsmethoden (industrielle Prozessmodellierungsmethoden werden aus Sicht der Sprach- und Kommunikationswissenschaft im BMBF-geförderten Projekt Interdisziplinäre Methoden industrieller Prozessmodellierung (IMIP) untersucht. Das Projekt wird vom Lehr- und Forschungsgebiet Textlinguistik/ Technikkommunikation, dem Institut für Arbeitswissenschaft und der Psychologie (Communication Science) der RWTH Aachen bearbeitet. Teile der erhobenen Daten werden dankenswerter Weise von Univ.-Prof. Dr. phil. Eva-Maria Jakobs für die Analyse der angestrebten Dissertation zur Verfügung gestellt). Industrielle Prozessmodellierungsmethoden stammen aus der Ingenieur- und Betriebswissenschaft. Sie werden in Unternehmen angewendet, um Geschäftsprozesse zu optimieren. Grob lässt sich die Methodenanwendung in zwei Schritte differenzieren. In einem ersten Schritt werden in mündlichen Interviews Unternehmensmitarbeiter über einen ausgewählten Geschäftsprozess befragt (Prozessaufnahme). Die Interviewer sind meist externe Berater; sie interessieren sich u.a. für Arbeitsabläufe, verwendete Ressourcen sowie Schwachstellen im Arbeitsablauf. In einem zweiten Schritt wird das erhobene Wissen der Mitarbeiter schriftlich und visuell in einem Prozessmodell abgebildet (Prozessmodellierung). Anhand des Prozessmodells werden Schwachstellen im Arbeitsprozess systematisch analysiert und Verbesserungsmaßnahmen für den Arbeitsprozess abgeleitet (Jakobs/Spanke im Druck).

Im Beitrag werden ausgewählte Ergebnisse einer diskursanalytischen Untersuchung zu Verständigungsproblemen in der Prozessaufnahme vorgestellt (der Begriff Diskursanalyse wird als Oberbegriff für verschiedene Traditionen (u.a. Gesprächs-, Konversations-, Dialog-, Kommunikationsanalyse/ -forschung/-linguistik) verwendet, die sich mit der Erforschung sprachlich vermittelter Kommunikation und Interaktion befassen (Becker Mrotzek/Meier 2002). Erste Ergebnisse zeigen, dass die Ursachen für Verständigungsprobleme vielfältig sind: Verständigungsprobleme entstehen beispielsweise, weil Kommunikationsteilnehmer zwar denselben sprachlichen Ausdruck verwenden, aber Unterschiedliches damit meinen oder weil Kommunikationsteilnehmer unterschiedliche Erwartungen an die Durchführung oder das Ziel der gemeinsamen Kommunikation richten.

Der Beitrag entsteht im Rahmen meiner angestrebten Dissertation. Ziel der Dissertation ist es, in der Prozessaufnahme auftretende Verständigungsprobleme und zugehörige Problemlöseverfahren $\mathrm{zu}$ identifizieren, $z u$ analysieren, $z u$ beschreiben und $z u$ typologisieren. Aus sprach- und kommunikationswissenschaftlicher Sicht leistet die angestrebte Dissertation damit einen Beitrag zur wissenschaftlichen Dokumentation und Erforschung von Verständigungsproblemen in der Unternehmenskommunikation.

\section{Literatur}

Becker Mrotzek, M./Meier, C. (2002): Arbeitsweisen und Standardverfahren der Angewandten Diskursforschung. In: Brünner, G./Fiehler, R./Kindt, W. (Hrsg.): Angewandte 
Diskursforschung. Band 1: Grundlagen und Beispielanalysen. Radolfzell: Verlag für Gesprächsforschung, 18-45

Fiehler, R. (Hrsg.) (2002): Verständigungsprobleme und gestörte Kommunikation. Radolfzell: Verlag für Gesprächsforschung

Jakobs, E.-M./Spanke, J. (im Druck): Sprache als Erfolgsfaktor industrieller Prozessmodellierung. Beitrag zur Konferenz "Europäische Kulturen zur Wirtschaftskommunikation" (EUKO), 26.-28. August, Winterthur, Schweiz. Wiesbaden VS Verlag

Kindt, W./Weingarten, R. (1984): Verständigungsprobleme. In: Deutsche Sprache. Zeitschrift für Theorie, Praxis, Dokumentation, 3, 193-218

Selting, M. (1987): Verständigungsprobleme. Eine empirische Annaylse am Beispiel der Bürger-Verwaltungs-Kommunikation. Tübingen: Niemeyer

\section{Ingmar Rothe}

Strikt neben dem Leitfaden. Analysen skriptbasierter Telefonie

Seit es Callcenter gibt, interessiert sich die (linguistische) Forschung für sie, von Anfang an standen auch Leitfäden im Fokus des vor allem kritischen Zugriffs. Die Erkenntnisse in Bezug auf Leitfäden sprechen eine recht deutliche Sprache: situativ per se unangemessen, weil zu starr, vergleichbar mit einem sprachlichen Korsett, das die negativen Aspekte der Emotionsarbeit noch verstärkt, unauthentisch, da leicht als vorgelesen identifizierbar. Basis des Beitrags soll meine im Juli 2009 abgeschlossene Diplomarbeit (Sprachliche und sprecherische Orientierung im skriptbasierten Verkauf am Telefon) sein, in deren Rahmen ein großes Gesprächskorpus (360 Gespräche) erschlossen wurde. Fünf Gespräche werden in der Arbeit im Sinne einer Kollektion typischer Fälle exemplarisch analysiert. Im AnalyseFokus steht vor allem der Vergleich reproduzierter, also vorgelesener, Sequenzen mit frei formulierten Sequenzen. Ausgehend von den Ergebnissen der Analyse dieser authentischen Gespräche mittels sprechwissenschaftlicher und gesprächsanalytischer Methoden möchte ich Ideen und Möglichkeiten einer Anschlussuntersuchung diskutieren. Im Mittelpunkt sollen dabei die Relevanz von Leitfäden in der aktuellen Callcenter-Praxis und Anwendungsbeispiele für die bereits vorliegenden Ergebnisse stehen.

\section{Milan Mihajlovic}

Jugend debattiert - a pragma-dialectic study

While trying to combine normative and descriptive elements, the pragma-dialectical approach to argumentation is mainly normative. This becomes very plain when one looks at the framework of rules set in order to promote the aim of resolving a difference of opinion in a rational way. Unlike traditional approaches to argumentation, these rules are not restricted to logical questions. They also refer to the conduct of participants in a verbal exchange. Given that the idealized pragma-dialectical approach provides a framework for rational resolutions of disputes, one interesting question that arises: where in social reality do we find such behavior as suggested by the rules? Or put differently: how far away is argumentative reality from that idealized conception? The Jugend debattiert contest, which is a debating contest in a professsionalized setting, may yield some empirical answers to this question. Besides, theoretical questions concerning the consistency and overall practicability of pragma-dialectics shall not be left out in my presentation (which may be held in English or in German). 


\section{Gabriella Carobbio}

„Wie Sie sehen können...”. Einwirkungen multimedialer Präsentationen auf Vorträge

Im Rahmen der Wissenschaftssprachforschung nehmen Studien zu den mündlichen Formen der Wissenschaftskommunikation an Bedeutung zu. Untersucht wird dabei oft die Diskursart „Wissenschaftlicher Vortrag“, die im Hinblick auf ihre konstitutiven Bestandteile und die sprachlichen Mittel zur Redeorganisation und zur Steuerung der Hörerrezeption beschrieben wird (Ventola u.a. 2002; Ylönen 2003; Hohenstein 2006; Debes 2007, Carobbio, im Druck).

Im heutigen technologischen Zeitalter werden Vorträge immer häufiger durch multimediale Präsentationen begleitet und unterstützt. Der Einsatz multimedialer Mittel, die zur Verständlichkeit des Diskurses beim Publikum beitragen sollen, erfordert seitens des Vortragenden eine "Synchronisierungsfähigkeit“, die laufende Rede mit den auf den Slides visualisierten Inhalten zusammenzubringen.

Anhand eines transkribierten Korpus von wissenschaftlichen Vorträgen soll der Frage nachgegangen werden, wie multimediale Technologien auf "klassische“ Vortragspräsentationen einwirken, auch im Hinblick auf die sprachlichen Verfahren, die bei der sprecherseitigen Bezugnahme auf Slides und Folien in Anspruch genommen werden. Der Beitrag soll damit spezifisch auf Aspekte der Multimedialität in der mündlichen Wissenschaftskommunikation eingehen.

\section{Literaturangaben}

Carobbio, Gabriella (im Druck) Autokommentierendes Handeln in der Wissenschaftssprache. Überlegungen zu einer möglichen Eingliederung in das sprachliche Handlungsmustersystem. In: Hornung, A. (Hg.) Lingue di Cultura in pericolo - bedrohte Wissenschaftssprachen. L'italiano e il tedesco di fronte alla sfida dell'internazionalizzazione/ Italienisch und Deutsch vor den Herausforderungen der Internationalisierung. Tübingen: Stauffenburg, 165-180.

Debes, Cornelia (2007) Mündlicher Wissenschaftsdiskurs Russisch-Deutsch. Ein sprachwissenschaftlicher Vergleich. In: DaF 44/1, 32-36.

Hohenstein, Christiane (2006) Erklärendes Handeln im wissenschaftlichen Vortrag: ein Vergleich des Deutschen mit dem Japanischen. München: iudicium.

Ventola, Eija / Shalom, Celia / Thompson, Susan (Hgg.) (2002) The Language of Conferencing. Frankfurt a.M.: Lang.

Ylönen, Sabine (2003) Konferenzdeutsch Unterschiede mündlicher und schriftlicher Wissenschaftssprache. In: Zhu, J. / Zimmer, T. (Hgg.) Fachsprachenlinguistik, Fachsprachendidaktik und interkulturelle Kommunikation. Frankfurt a.M.: Lang, 209-229.

\section{Jan Gerwinski}

Medienvermittelte Ortserkundung in multilokalen und multimodalen Interaktionsräumen am Beispiel von Feuerwehreinsatzübungen

Im Kontext von Notfall-Feuerwehreinsätzen ist für die beteiligten Einsatzkräfte eine Vielzahl raumbezogener Kommunikationsaufgaben mediengestützt zu bearbeiten. Dabei werden u.a. Funkgeräte, Einsatzpläne, ggf. Karten/ Lagepläne, Wärmebildkameras und neue technische Steuerungs-, Lokalisierungs- und Referenzierungs-Hilfen verwendet. Insgesamt ist die Interaktion auf dieser Basis durch Multimodalität charakterisiert. Diese Multimodalität untersuche ich exemplarisch an einem Spezialfall, denn ich erhebe meine Daten in 
Kooperation mit einem Forschungsprogramm, in welchem neuartige technische Medien zur Koordination und Navigation der Feuerwehreinsatzkräfte entwickelt werden (sog. „Landmarken“; siehe www.landmarke-projekt.de). Ein weiteres Charakteristikum ist die Multilokalität der Kommunikation, da sich die unterschiedlichen Einsatztrupps untereinander und v.a. mit dem Einsatzleiter koordinieren müssen. Bzgl. dieser beiden Spezifika können - speziell im Bereich der Raumrepräsentation - Pannen und Krisen zweiter Ordnung auftreten, die die Beteiligten im Hinblick auf das gemeinschaftliche außersprachliche Primärziel wiederum kommunikativ lösen müssen. Anhand der damit verbundenen Artikulationen werden in meinem Forschungsprojekt spezifische Probleme dieser multilokalen und multimodalen Kommunikation mit Hilfe authentischen, empirisch erhobenen (audiovisuellen) Datenmaterials aus linguistischer Perspektive analysiert, wobei insbesondere Räume - ihr Einfluss auf sowie ihre sprachlich-mediale Repräsentation im Kontext - fokussiert werden.

Meine leitenden Forschungsfragen lauten dabei:

1. Welchen Einfluss nehmen neue technische Medien auf die Kommunikation in arbeitsteiligen Prozessen hinsichtlich (Zeichen-)Raumkonstitution und Navigation? Dabei liegt ein besonderer Fokus auf der Frage, ob eine mögliche Transparenz der Medien hilfreich und wünschenswert wäre.

2. Wie und wozu erfolgt in diesem Rahmen die Lokalisierung, sowohl der beteiligten Personen als auch elementarer Objekte?

3. Wie erfolgt die interaktionale Steuerung der beteiligten Personen im Raum?

Wesentliche theoretische Grundlagen für die Untersuchung bilden Konzepte der Raumlinguistik und neuere linguistische Untersuchungen zu multimodalen Koordinationsprozessen. Das methodische Vorgehen bei der Datenerhebung und -analyse orientiert sich an Prinzipien der Ethnomethodologischen Konversationsanalyse sowie den daraus hervorgegangenen Workplace Studies bzw. Studies of Work.

\section{Section I Language Development}

\section{Nicole Riechert}

Die (neuen) Stimmen der Jugend: Über die Signifikanz der deutschen Rapmusik

Nicht selten werden Künstler, wie der deutsche „Rüpel-Rapper“ Bushido, wegen ihrer Wortwahl und Inhalte kritisiert oder gar verboten. Stimmen aus der Politik, Pädagogik, Medienlandschaft oder anderen gesellschaftlichen Bewegungen verurteilen diese Jugendkultur scharf. Doch was ist das Geheimnis ihres Erfolges? Eine Betrachtung dieses kulturellen Mediums wirft diverse Kontroversen auf: Wollen die Künstler mittels ihrer Musik durch biografische Berichte aus ghettoisierten, sozialen Brennpunkten auf Missstände in der Gesellschaft aufmerksam machen, vermitteln sie die „neuen Werte“ der Jugend oder ist das „Böse Bube“-Image doch nur eine kalkulierte Geschäftsidee, um mittels einer Marke ein „Wir-Gefühl“ zu erwecken? Will man also von den „Stimmen der Jugend“ sprechen, müssen gesellschaftliche Gegebenheit und der Lebenskontext in eine Untersuchung einbezogen werden. Häufiger Diskussionsgegenstand und umso kontroverser sind Inhalte, die als schwulen- und frauenverachtend sowie sexistisch und gewaltverherrlichend interpretiert 
werden können. In der Arbeit über „die (neuen) Stimmen der Jugend“ wird nach den verschiedenen Einflüssen des deutschsprachigen Hip-Hops als polarisierende Jugendkultur gefragt, obgleich sich die theoretische und empirische Ausrichtung auf den Einfluss und die Macht der Künstler in Bezug auf die Jugendlichen als Rezipienten beschränken. Referenzpunkte der Untersuchung sind die Bedeutungen und Symbole in der Musik, um im Anschluss die Relevanz dieser medialen Aufarbeitung in Hinblick auf einen Machteinfluss zu untersuchen. Die Arbeit stellt sich die Aufgabe nach der Macht der Worte in der deutschsprachigen Rapmusik zu fragen. Es soll eruiert werden, worauf diese Macht begründet ist, wen sie erreichen kann und was man durch sie „bewegen“ kann und will.

Hierbei wird nicht allein aufgezeigt, welches Gedankengut wie vermittelt wird, sondern überdies ein Zusammenhang zwischen diesem, den Akteuren und dem Kontext der kommunikativen Handlung hergestellt. Ziel ist, den strategischen Einsatz von Kommunikation anhand der Signifikanz der deutschen Rapmusik zu erklären. Hierfür werden die Deutungswelten sowie Kollektivsymboliken dieser Jugendkultur dargestellt. Ebenso wird eruiert auf welche Art und Weise diese Musik rezipiert wird.

\section{Christian Ludwig}

What Comics can do: The Use of Graphic Fiction in SLA-Processes and the Development of Visual Literacy

This paper aims to show how (alternative) comics can be put to use in the foreign language classroom for various purposes. Comics have long been regarded as no suitable subjects for scholarly pursuit. The notion that they trigger aggressive behaviour in young teenagers has largely barred them from the language classroom. Although, admittedly, the reputation of graphic fiction has improved since the advent of the graphic novel in the 1970s - Spiegelman being awarded the Pulitzer Prize for his Maus is a case in point -- teachers are still reluctant to make use of comics, most of all because they undervalue the potential of the genre. However, one can observe a shift of paradigms in modern foreign language learning and teaching mainly due to the shift from rather instructivist to more constructivist approaches. Moreover, visual literacy is more and more regarded as indispensable to education and daily life. Within this context comics can play an important role. They present a hybrid form of discourse that combines pictures with oral and written language and offer vital connections between literature and the learners' life. By providing access to authentic language they connect the classroom with the outside world. Apart from being culturally loaded in terms of content they also represent a significant part of the material expression of culture. Additionally, comics offer the non-verbal context of communication which is often absent in everyday classroom communication. To sum up, it can be said that graphic fiction has got the potential to initiate contextualised intercultural communication processes that enable and support second language learning. Additionally, the "act of reading a comic cuts much more closely to how our students today receive information" (Versaci, 1997) even though the dual coding of graphic fiction requires certain "reading" competencies in order to apprehend the narrative content and discover the deeper meaning of the narrative.

By analyzing recent underground comics (e.g. Julie Doucet's My New York Diary or Adrian Tomine's Summer Blonde) I will not only show that comics are a worthwhile subject matter in their own right but also that they can sensibly be employed for language teaching, i.e. the acquisition of linguistic and communication skills in the target language. Graphic fiction 
actively involves learners into the language learning process and at the same time enables them to acquire the foreign language more autonomously and successfully. Within this context, the focus will be on the specific linguistic characteristics and purposes of graphic fiction which will be presented in the context of current approaches to second language acquisition.

\section{Jana Gamper \\ "Der Junge zeigt den Polizist die Zunge» - Kasusmarkierungen in Texten von DaZ-Lernern}

Der Erwerb der deutschen Nominalflexion stellt für Lerner des Deutschen als Zweitsprache eine große Herausforderung dar. Während zum Beispiel Kinder die Syntax des Deutschen relativ früh zielsprachenkonform anwenden (Kaltenbacher \& Klages 2006), bleibt die normgerechte Markierung des Kasus oft problematisch (Grießhaber 2006, Sahel 2010, Mehmel 2004).

Dies lässt sich zum einen auf die Komplexität des deutschen Kasussystems zurückführen. Genus, Numerus und Kasus sind im Deutschen interdependet. Die Verwendung einer Kasusmarkierung muss demnach kongruent zum Genus und Numerus des entsprechenden Nomens sein. Gleichzeitig findet eine Markierung des Kasus nur in Einzelfällen am Nomen selbst statt. Vielmehr werden Adjektive, Artikel oder Pronomina hinsichtlich des Kasus gekennzeichnet, was vor allem bei der Verwendung komplexer Phrasen eine hohe kognitive Leistung erfordert. Zudem sind die Kasusflexive des Deutschen multifunktional und unterliegen einem hohen Grad von Synkretismus.

Zum anderen lassen sich spracherwerbstheoretische Gründe anführen. Studien zum Kasuserwerb durch Kinder mit deutscher Erstsprache haben gezeigt, dass die Verwendung von Kasusmarkierungen im Vergleich zu anderen Sprachen wie Russisch oder Türkisch später einsetzt. Es kann demnach davon ausgegangen werden, dass die entsprechende sprachinterne Komplexität des Flexionssystems auch Auswirkungen auf den Erwerb des Kasussystems durch L2-Lerner hat (Tracy 1984).

Vor dem Hintergrund dieser Überlegungen möchte ich in meinem Beitrag auf die Verwendung von Kasusmarkierungen in schriftlichen Texten von Grundschulkindern eingehen. Die Texte wurden im Jahr 2010 im Rahmen des Modellprojekts «Chancen der Vielfalt nutzen lernen» in den Jahrgangsstufen zwei bis vier an ausgewählten Münsteraner Grundschulen erhoben. Es soll unter anderem gezeigt werden, welche Kasus in welchen Kontexten verwendet und welche Konstituenten der Nominalphrase hinsichtlich der Kasus markiert werden. Der Fokus des Beitrags liegt dabei auf der Identifizierung von Problemen, die die Kinder bei der normkonformen Verwendung der deutschen Kasusmarkierungen haben und auf der Formulierung möglicher Implikation für den Umgang mit diesen Problemen im Zweitsprachenunterricht in der Grundschule.

\section{Literatur (Auswahl):}

Bittner, Dagmar (2004): Case Before Gender in the Acquisition of German. In: Folia Linguistics $\mathrm{XL}, 115-134$.

Griesshaber, Wilhelm (2006): Die Entwicklung der Grammatik in Texten vom 1. bis zum 4. Schuljahr. Bernt Ahrenholz (Hrsg.): Kinder mit Migrationshintergrund. Spracherwerb und Fördermöglichkeiten. Freiburg im Breisgau: Fillibach Verlag, 150-167.

Kaltenbacher, Erika \& Klages, Hana (2006): Sprachprofil \& Sprachförderung bei Vorschulkindern mit Migrationshintergrund. In: Bernt Ahrenholz (Hrsg.): Kinder mit 
Migrationshintergrund. Spracherwerb und Fördermöglichkeiten. Freiburg im Breisgau: Fillibach Verlag, 80-97.

Mehmel, Ulrich (2004): Kasusmarkierungen in Verschriftungen mündlicher Nacherzählungen bei marokkanischen Migrantenkindern. In: Ursula Bredel, Gesa Siebert-Ott \& Tobias Thelen (Hrsg.). Schriftspracherwerb und Orthographie. Baltmannsweiler: Schneider Verlag Hohengehren, 162-188.

Ritterbusch, Rachel; LaFond; Larry \& Agustin, Marcus (2006): Learner Difficulties with German Case: Implications from an Action Research Study. In: Die Unterrichtspraxis / Teaching German 39, 30-45.

Sahel, Said (2010): Ein Kompetenzstufenmodell für die Nominalphrasenflexion im Erst- und Zweitspracherwerb. In: Ulrich Mehlem \& Said Sahel (Hrsg.): Erwerb schriftsprachlicher Kompetenzen im DaZ-Kontext: Diagnose und Förderung. Freiburg im Breisgau: Fillibach Verlag, 185-209.

Tracy, Rosemarie (1984): Fallstudie: Überlegungen zum Erwerb von Kasuskategorie und Kasusmarkierung. In: Hartmut Czepluch \& Hero Janßen (Hrsg.): Syntaktische Struktur und Kasusrelation. Tübingen: Gunter Narr Verlag, 271-313.

\section{Margit Hempel}

Englisch Lernen als Kontinuum - Entwicklung von produktiven Fähigkeiten und Fertigkeiten beim Übergang von der Primarstufe in die Sekundarstufe

Trotz erheblicher Anstrengungen der Primarschulen wie auch der Sekundarschulen ist die Anschlussfähigkeit beim Übergang im Englischunterricht von der Primarstufe in die Sekundarstufe noch nicht hinreichend gewährleistet (Burwitzer-Melzer/Legutke 2004, Doms 2010). Die Evening Studie 2005 - 08 z.B. bescheinigte den Grundschülern in NRW hohe Kompetenzen im Bereich „Hör- und Hör-Sehverstehen“, während die Leistungen im Bereich des produktiven Sprechens den Anforderungen, die sich am Gemeinsamen Europäischer Referenzrahmen für Sprachen (GER) orientieren und somit dem von der Sekundarstufe I erwarteten Abschlussprofil, oft nicht entsprachen (Engel 2009, Doms 2010, Diehr/Rymarcyk 2008).

Vor dem Hintergrund empirischer Erhebungen in der Primarstufe möchte ich in meinem Beitrag auf die Spezifika von interaktiven Sprechakten in der Fremdsprache (L2) und den Einfluss von Kontextfaktoren auf den kognitiven Erwerbsprozess und darauf basierend auf die Hinführung vom imitativen/reproduktiven zum produktiven/konstruierenden/interaktiven Sprechen eingehen.

Ein Fokus liegt dabei auf den in der Primarstufe wie auch in der Sekundarstufe I verwendeten Lehrwerken. Als Ausblick soll eine mögliche Intervention in beiden Schulformen zur Verbesserung von L2 Sprachfertigkeiten und Herstellung der Anschlussfähigkeit gegeben werden.

Literatur:

Burwitzer-Melzer, Eva/Legutke, Michael (2004): Die Übergangsproblematik. In: Der fremdsprachliche Unterricht Heft 69

Diehr, Bärbel/Rymarczyk, Jutta (2008): „Ich weiß es, weil ich es so spreche“. In: Grundschulmagazin Englisch 1

Doms, Christiane (2010): Lernstand diagnostizieren. In: Der fremdsprachliche Unterricht 103 
Doms, Christiane (2010): Lost in transition? Herausforderungen des Übergangs bewältigen. In: Grundschulmagazin Englisch 4

Engel, Gaby/Groot-Wilken, Bernd/Thürmann, Eike (Hrsg.) (2009): Englisch in der Primarstufe - Chancen und Herausforderungen. Berlin: Cornelsen

Pienemann, Manfred/ Keßler Jörg-U./RoosEckhard (Hrsg.) (2006): Englischerwerb in der Grundschule. Paderborn: UTB

Wagner, Ute (2009), Übergang Englisch. Tübingen: gnv

Roos, Jana (2007): Spracherwerb und Sprachproduktion. Tübingen: gnv

\section{Hana Smiskova}

Conventionalized sequences in $L 2$ development

This paper reports on a longitudinal study of L2 English development from a usage-based perspective. A group of 20 Dutch high school learners in high-input (bilingual education, TTO) and low-input conditions (regular education, gymnazium) were followed over 2.5 years, with a focus on potential differences in L2 developmental paths. Preliminary studies in our subject population had shown that such differences may be best captured by tracking the learners` use of formulaic sequences (Wray 2002).

We took an integrated approach to the definition and identification of formulaic sequences (Granger 2008, Wray 2008) in written samples of our learners' language. A combination of phraseological and frequency-distributional analyses captured significant differences between the high and low input groups (Smiskova \& Verspoor, forthcoming). These concerned mainly formulaic sequences which do not easily fit any prescribed phraseological categories: conventionalized sequences of three and more words structurally equal to phrases, clauses and sentences of various degrees of complexity (eg. The only thing I know for sure is that ...). At the same time, our study has revealed commonly recognized methodological problems, such as vague borders or overlaps in formulaic sequences (eg. [[The only thing I[know [for sure]]] is that]...) and difficulties with reliable identification and categorization of formulaic sequences.

Such findings are in line with usage-based approaches, which regard language as a continuum of constructions with no rigid separation between lexicon and grammar (Ellis \& Cadierno, 2009). During their L2 development, learners need to build a vast repertoire of constructions at different levels, including conventionalized, "normal ways of saying things" (Langacker, 2008). Building on the findings and methodological challenges revealed during our study of formulaic sequences and using detailed examples of the learners 'language, this paper explores a possible application of the cognitive-constructionist approach to our further study of L2 development in our subject population.

\section{References:}

Ellis, N. C. \& Cadierno, T. (2009). Constructing a Second Language: Introduction to the Special Section. Annual Review of Cognitive Linguistics, 7, 111-139.

Granger, S. \& Paquot, M. (2008). Disentangling the phraseological web. In S.Granger \& F. Meunier (Eds.), Phraseology: An interdiciplinary perspective (pp. 28-49). Amsterdam: John Benjamins.

Langacker, R. (2008). Cognitive Grammar and Language Instruction, in Robinson, P. \& Ellis, N. (eds.), Handbook of Cognitive Linguistics and Second Language Acquisition. Routledge: New York and London.) 
Smiskova \& Verspoor, forthcoming. Development of Formulaic Sequences in Dutch L2 Learners of English. In Evers-Vermeul, J., Rasier, L., \& Tribushinina, E. (eds). Usage-Based Approaches to Language Acquisition and Language Teaching (series Applications of Cognitive Linguistics), Mouton de Gruyter.

Wray, A. (2002). Formulaic Language and the Lexicon. Cambridge: Cambridge University Press.

Wray, A. (2008). Formulaic language: Pushing the boundaries. Cambridge: Cambridge University Press. 


\section{Dorina Veldhuis, Ad Backus}

Chunks for segmentation and processing

There is a discrepancy between people's beliefs on what counts as the basic building blocks of language, and the current view of many linguists. Most laypeople nowadays suppose, as some early studies into children's metalinguistic development suggested, that words are the basic units of language segmentation. This view is primarily informed by schooling in languages with alphabetic writing systems in which words are marked, and by the pervasive role of written language in the lives of most of us and extensive number of linguistic studies in such languages. Nevertheless, developments in linguistic theory and empirical findings in psycholinguistics suggest that, deep down, the units which we combine to build utterances are not, generally, single words. Evidence suggests that words often combine into larger units or chunks, which are presumably the real basic units of language. This discrepancy between the views of the layperson and of the linguist raises several questions. First, if units larger than words are the building blocks, then which combinations of words can be found in such larger chunks? Secondly, what are the restrictions on and the requirements for the development of such chunks? How and when do multi-word chunks get so entrenched that they are segmented and processed as wholes, and what evidence can we find for this? For the investigation of these questions, we conducted three offline segmentation experiments as well as three online language perception and language production experiments among 204 Dutch children. Our offline results showed that frequency of occurrence and salience mainly affect pre-literate children's segmentation units; the online tasks showed how frequency and salience affect the way in which multi-word chunks are unconsciously processed.

\section{Section II Language and Community in Media}

\section{Jude Ssempuuma}

The Role of English in Ugandan Political Debates: Hedges and Rhetoric Questions in Political Campaigns in Kampala.

In Uganda, English was introduced in the education system by the missionaries and British administration at an earlier stage during the colonial period. Its use in public discourse was limited since the British preferred using a local lingua franca. Luganda therefore was used as lingua franca during the British indirect rule in Buganda and other parts of the country such as Busoga, Bugisu and Kigezi as the language of administration during the colonial period (Thomas et al. 1949: 89, Richards 1969: 10). Though spoken by a small elite group, English was declared the official language of Uganda at independence in 1962. However, Luganda continued to be the mostly widely spoken language in the country (Ladefoged et al. 1972, Mukama 1991, Kasozi 2000, Nsibambi 2000).

Since the 1990s, the National Resistance Movement (NRM) government has made policies which have promoted English and thus increased the number of English speakers in the country. For instance, in the urban areas, English is the only language of instruction from primary one and throughout all education levels. In addition, the Universal Primary Education (UPE) and Universal Secondary Education (USE) government policies have led to an increase in the number of school attendants who use English as a second language. In 
Kampala, the capital city of the country, English is becoming the dominant language of the media, entertainment and public discourse replacing Luganda, the widely spoken local language and the language of the biggest ethnic community in the country and in the capital city. For instance, during the Buganda Annual Conference in 2009 and 2010, English rather than Luganda was used. Although the conference is organised by the Buganda kingdom, topics discussed concerns both Buganda and Uganda at large. Furthermore, speakers at the conference come from different parts of Uganda and the world, a factor that necessitates the use of English language.

This presentation will discuss which factors support English use in urban public discourse, especially in political campaigns. For instance, English as a neutral language in a multilingual urban Ugandan community most especially in Kampala. It will illustrate discourse features of Ugandan English such as hedges and rhetoric questions.

\section{References}

Ladefoged, Peter, Ruth Glick, and Clive Criper (eds.) 1972. Language in Uganda. London: Oxford University Press.

Mukama, Ruth G.1991. "Getting Ugandans to speak a common language: Recent developments in the language situation and prospects for the future" In: Hansen Holger Bernt and Twaddle Michael (eds.), Changing Uganda: The Dilemmas of Structural Adjustment and Revolutionary Change. Kampala: Fountain Press. $334-350$

Nsibambi, Apolo. 2000. "Language and literacy in Uganda: A view from the Ministry of Education and Sports" In: Parry, Kate (ed.), Language and Literacy in Uganda.

Kampala: Fountain Publishers Ltd. 2-5.

Richards, Audrey. 1969. The Multicultural States of East Africa. Montreal: McGill-Queen's University Press.

Thomas H. B, O. B. E and Robert Scott (eds.) 1949. Uganda. London: Oxford University Press.

\section{Anna Tkachenko}

"Ich bin ein stolzer Mixer zwischen Russe und Deutscher": Versprachlichungsstrategien russischer Migranten im Chat

Russische Migranten verwenden in bilingualen Chatkanälen beide Sprachen abwechselnd. Bei der Schriftauswahl ist man auch relativ flexibel: Man schreibt sehr oft deutsch in kyrillischer und russisch in lateinischer Schrift. Die Transliteration unterliegt auf den ersten Blick keinen Regeln. Beim näheren Betrachten werden jedoch die orthographischen Einflüsse aus beiden Sprachen sichtbar. In einigen Fällen werden deutsche Wörter in kyrillischer Schrift so transkribiert, wie sie von Sprachlernern in Analogie zur Phonetik der Muttersprache wahrgenommen werden. Diese "Laien-Transkription" dient meistens als Stilmittel, um der Aussage einen umgangssprachlichen Charakter zu verleihen. Häufig tritt auch das Code-Switching auf, das nicht selten innerhalb einer Äußerung erfolgt, wobei die Schrift aus Zeitmangel nicht gewechselt wird. In dem Vortrag sollen diese und andere Phänomene zu Versprachlichungsstrategien russischer Migranten im Chat ausgeführt werden. 


\section{Julia Wrede \\ Bedeutungs-(Re-)Konstruktion durch den Sprachgebrauch: Eine korpuslinguistische Untersuchung kognitiver Phänomene und semantischer Konsequenzen}

Kognitionslinguisten verstehen unter der Bedeutung eines Wortes üblicherweise die mit ihm verknüpfte mentale Repräsentation und vertreten häufig eine konzeptprioritäre Sprachauffassung. Doch nimmt Sprache keinerlei Einfluss auf den Konzeptaufbau? Für Korpuslinguisten wie z. B. WOLFGANG TEUBERT sind konzeptuelle Strukturen wolkige Konstrukte, seiner Meinung nach erhielten natürlichsprachliche Symbole ihre Bedeutung einzig dadurch, dass sie im Diskurs gebraucht würden. Er regt dementsprechend an, Konzepte als den gemeinsamen Besitz einer Diskursgemeinschaft zu verstehen, sodass sie nicht notwendigerweise in den Köpfen der Menschen gesucht werden müssten (vgl. TEUBERT 2006: 291).

Ihm zufolge könnten mentale Konzepte nicht mehr, als das zu duplizieren, was sich ohnehin schon im Sprachgebrauch finden ließe (vgl. TEUBERT 2006: 296). Ziel des Vortrags soll sein, in Ansätzen zu zeigen, wie sich diese vermeintliche Dublette des Sprachgebrauchs aufgrund eben des Sprachgebrauchs aufbaut, strukturiert, wandelt und variabel ist sowie fortlaufend in ihrer Bedeutung implizit in der Kommunikation fortwirkt. Wir wollen also durchaus ein Konzept hinter einem Ausdruck annehmen, das jedoch von der Kotextualisierung des Ausdrucks abhängt (die als Etablierung einer Framestruktur und Auffüllung der FrameDimensionen mit Werten sowie davon abhängender kognitiver Standardwert-Etablierung semantiktheoretisch verortet werden kann (vgl. ZIEM 2008). Am Beispiel der sprachlichen Einheit [der] 11. September soll - anhand der Arbeit mit Textkorpora (die Korpora des Instituts für Deutsche Sprache in Mannheim, die mit der Analysesoftware COSMAS II bearbeitet werden können (http://www.ids-mannheim.de/cosmas2/) -veranschaulicht werden, dass und wie wir unser diesem Ausdruck zugehöriges Wissen aus medialer Vermittlung in Texten aufgebaut haben und wie sich die konzeptuelleWissensstruktur des Ausdrucks (Sowie weiterer sprachlicher Elemente aus dem Diskurs (z. B. Anschlag, Ereignis)aufgrund der fortlaufenden usuellen, aber auch variablen Kotextualisierung konstituiert und entwickelt hat.

Doch es ist nicht nur die anwendungsbezogene Korpuslinguistik, die der Flexibilität und Dynamik von Bedeutung Rechnung trägt. Frames als semantiktheoretische Größen der strukturellen Form von Bedeutungspotentialen lassen sich mit korpusanalytischer Methodik verknüpfen. Die von TEUBERT (vgl. 2007: 23) beschriebene Hinzufügung von neuem semantischen Wissen durch neue Diskursbelege erinnert an die framesemantischen Größen der Füllwerte in der Kotextualisierung, die sich zu kognitiv verfestigten Standardwerten aus dem Sprachgebrauch via Usualität entwickeln können, die wiederum in der weiteren Kommunikation über ihre Implikation semantisch fortwirken. Die Füllwerte sind Korpusdaten zu entnehmen, sodass innerhalb der hier vorgestellten Arbeit die beiden Bereiche der Korpuslinguistik als Anwendungsdisziplin und der Kognitiven Linguistik als Sprachtheorie verbunden werden sollen: empirische Daten aus Korpora stellen dabei die Grundlage für eine angedachte Theoriemodifikation dar, dass die Bedeutung sprachlicher Einheiten von den sie umgebenden Diskurselementen abhängt. Konzepte sind als kollektive Entitäten über ihren Ursprung in der kollektiven Sprachverwendung zu deuten.

Die Arbeit reiht sich somit in eine neue Tradition ,Kognitiver Linguistik 2.0 - so möchte ich sie nennen - ein, die sprachgebrauchsorientiert ansetzt und in Sprache die Basis für den Konzeptaufbau sieht. Statt Sprache lediglich als Reflexion konzeptueller Strukturen zu 
erachten, wird ihr als konstitutivem Faktor für diese Geltungsrecht eingeräumt. Es soll gezeigt werden, dass Konzepte von Wörtern aufgrund der Kotextualisierung der Wörter variabel sind. Korpusdaten können uns via Berechnungsverfahren als Darstellung usueller Kotextualisierungen aufbereitet werden, sodass wir hier in Form von überindividuellem Sprachgebrauch als angenommene Grundlage für überindividuell verankerten Konzeptaufbau (entrenchment) eine Datenbasis vorliegen haben. Die Arbeit berücksichtigt somit die Verknüpfung von individuellem Wissen, gesellschaftlich geteiltem Wissen und Wortgebrauch als individuelle und soziale Perspektive auf sprachliche Bedeutung, die damit nicht mehr unverbunden nebeneinander stehen.

\section{Literatur (in Auswahl):}

ALLWOOD, JENS 2003: Meaning potentials and context: Some consequences for the analysis of variation in meaning. In: CUYCKENS, HUBERT/DIRVEN, RENÉ/TAYLOR, JOHN R. (Hgg.) 2003: Cognitive Approaches to Lexical Semantics. Berlin: Mouton de Gruyter. S. 29-65.

CROFT, WILLIAM 2000: Explaining Language Change: An Evolutionary Approach. Harlow: Longman.

LINZ, ERIKA 2002: Indiskrete Semantik. Kognitive Linguistik und neurowissenschaftliche Theoriebildung. München: Fink.

LOPPE, TIM 2010: Bedeutungswissen und Wortgebrauch. Entwurf einer Semantik im Anschluss an Wittgenstein und Putnam. Tübingen: Narr.

TEUBERT, WOLFGANG 2006: Über den fragwürdigen Nutzen mentaler Konzepte. In: PROOST, KRISTEL/WINKLER, EDELTRAUT (Hgg.) 2006: Von Intentionalität zur Bedeutung konventionalisierter Zeichen. Tübingen: Narr. S. 289-325.

TEUBERT, WOLFGANG 2007: Korpuslinguistik, Hermeneutik und die soziale Konstruktion der Wirklichkeit (Teil 1). In: Sprachreport 3/2007. S. 19-24.

ZIEM, ALEXANDER 2008: Frames und sprachliches Wissen. Kognitive Aspekte der semantischen Kompetenz. Berlin u. a.: de Gruyter.

Julia Richling, Bianka Trevisan, Eugenie Diesbrecht, Michael Beißwenger

Korpusgestützte Analyse sprachlicher Besonderheiten in der internetbasierten Kommunikation: Brückenschläge zwischen Angewandter Linguistik und Maschineller Sprachverarbeitung

Der Vortrag skizziert am Beispiel zweier laufender Dissertationsprojekte zur internetbasierten Kommunikation (IBK) sowie anhand eines Forschungsprojekts zur automatischen Wortartenannotation, wie sich die Perspektiven von Angewandter Linguistik und Maschineller Sprachverarbeitung bei der Aufbereitung und Analyse von IBKSprachdaten wechselseitig ergänzen können.

Die drei Projekte kooperieren im Wissenschaftlichen Netzwerk „Empirische Erforschung internetbasierter Kommunikation“ (http://www.empirikom.net), das seit Ende 2010 von der Deutschen Forschungsgemeinschaft (DFG) gefördert wird. Nach einem Überblick über die Schwerpunkte des Netzwerks werden zunächst die einzelnen Projekte vorgestellt und methodische Desiderate formuliert, die sich bei der Bearbeitung der betreffenden Forschungsvorhaben ergeben. Davon ausgehend wird aufgezeigt, wie die Projekte durch eine wechselseitige Bereitstellung von Ressourcen voneinander profitieren können und werden Schnittstellen für die Zusammenarbeit formuliert. 
a) Das Dissertationsprojekt "Sprachwandel in Online-Foren" von Julia Richling untersucht die sprachliche Variation innerhalb von Forengemeinschaften im mikrodiachronen Verlauf. Die Kernfrage lautet, in welchem Rahmen die für eine Gemeinschaft typischen sprachlichen Muster die sprachlichen Gewohnheiten der agierenden Individuen beeinflussen und umgekehrt.

So gibt es in der schriftlichen IBK beispielsweise eine Reihe von Möglichkeiten, um Ironie zu markieren oder um andere Kommunikationsbeteiligte zu adressieren. Die Frage ist nun, inwieweit sich Schreiber beim Agieren in einer Forengemeinschaft an den in der Gemeinschaft vorherrschenden Präferenzen bezüglich der sprachlichen Realisierung dieser Funktionen orientieren, unter welchen Bedingungen sie von den Gewohnheiten abweichen und unter welchen Bedingungen individuelle Abweichungen von gebräuchlichen Mustern zu einer Veränderung der Gewohnheiten der Gemeinschaft führen können.

b) Das Dissertationsprojekt „Evaluation und Bewertung von Verfahren zur Erhebung sprachlicher Anteile von Bewertungsmustern" von Bianka Trevisan beschäftigt sich mit der kriterienbasierten Evaluation von Methoden und Techniken zur Erhebung von sprachlichen Bewertungsmustern (pos/neg-Ausdrücke, Referenzierungen) aus kommunikationswissenschaftlicher Sicht. Ziel des Dissertationsvorhabens ist die Entwicklung einer Methodik zur Erhebung sprachlicher Anteile von Bewertungsmustern, unter anderem mit Blick auf Nutzerbewertungen in Social Media Tools.

Fokussiert wird auf die Frage, wie sich anhand der Methode des Blog Mining (einer speziellen Form des Text Minings für Weblogs) in unstrukturierten IBK-Daten sprachliche Indikatoren für Themen und Bewertungsmuster identifizieren lassen und wie die dabei erzielten Ergebnisse für die linguistische Analyse (aber auch für andere Forschungsfelder) nutzbar gemacht werden können. Zur Veranschaulichung werden Beispiele aus dem assoziierten Projekt „Akzeptanzbewertung als integraler Bestandteil von Entwicklung und Ausbau komplexer technischer Systeme" (HUMIC, RWTH Aachen) herangezogen.

c) Das Projekt „Automatisches Part-of-speech-Tagging für die schriftliche internetbasierte Kommunikation" (Eugenie Giesbrecht / Stefan Evert) beschäftigt sich mit der Weiterentwicklung eines Werkzeugs zur automatischen Wortartenannotation (,Part-of-Speech-Tagging“, „POS-Tagging“) in Hinblick auf den Umgang mit Normabweichungen in schriftlicher IBK.

Die automatische Zuordnung bestimmter Textdatensegmente zu Wortartenkategorien ermöglicht es somit, Sprachdaten(korpora) um bestimmte grammatische Strukturinformationen anzureichern (wie z.B. „Verb“ oder „Nomen“ zu einzelnen Wortvorkommen) und der computergestützten linguistischen Analyse somit die Möglichkeit der Suche über Wortartenkategorien und Abfolgen/Mustern von Wortartenkategorien in den Daten zu eröffnen.

Bislang existierende Verfahren/Werkzeuge (sog. „POS-Tagger“) wurden v.a. auf Zeitungskorpora trainiert und evaluiert. Während die in der Literatur beschriebenen Genauigkeiten beim POS-Tagging für Zeitungstexte bei 97-98\% liegen, werden bei Texten aus dem WWW jedoch deutlich niedrigere Werte (90\%-92\%) erreicht. Dabei variiert die in dem Grad, in dem die auf WWW-Seiten vorfindliche schriftlichen Sprachverwendung von der Normorthographie abweicht. Insbesondere in solchen Nutzungskontexten von IBK-Technologien (z.B. Foren oder Weblogs), in denen informell kommuniziert wird, sind Normabweichungen hoch frequent (bedingt 
u.a. durch eine Orientierung an der gesprochenen Umgangssprache, eine kreative Verwendung von Satz- und Sonderezeichen oder Verfremdungsschreibungen). Ziel des Projekts ist es, die Qualität des Outputs eines als Komponente des Apache UIMA Frameworks entwickelten POS-Taggers 1 an die Besonderheiten informeller schriftlicher IBK anzupassen und als Tagger für IBK-Daten weiterzuentwickeln.

\section{Juliane Gall \\ Sprache im Alter - Der Einfluss sozialer Faktoren auf Sprachliche Veränderungen bei älteren Menschen}

Die zunehmende Alterung der Gesellschaft wurde in den letzten Jahren in verschiedenen Wissenschaftsdisziplinen untersucht. Im Bereich der Sprachwissenschaft jedoch scheint die Erforschung der Sprache unterschiedlicher Gesellschaftsgruppen das Alter auszuschließen, zumindest bleibt in der germanistischen Linguistik die Kommunikation älterer Menschen trotz intensiver Bemühungen einiger Wissenschaftler ein vernachlässigter Bereich.

Um altersbedingte Varianz zu erklären, werde ich im Rahmen meines Forschungsvorhabens die beeinflussenden Faktoren sprachlicher Veränderungen im Alter aufzeigen. Erst soll Alter definiert und seine Merkmale beschrieben werden. Im zweiten theoretischen Teil werden Begriffe wie Varietät, Gruppensprache und Stil erläutert, um zu zeigen, dass es schwierig ist, eine Definition für eine „Alterssprache“ zu finden. Dass sich sprachliche Veränderungen mit zunehmendem Alter vollziehen und wie diese aussehen, wird im dritten Teil anhand verschiedener Ansätze und ihrer Ergebnisse aufgearbeitet.

Im vierten Teil der Arbeit wird versucht, altersbedingte Varianz zu erklären. Eine theoretische Basis liefert der Korrelationsansatz, bei dem von einer systematischen Wechselbeziehung zwischen Alter und sprachlich-kommunikativen Phänomenen ausgegangen wird. Für die Ausprägungen der sprachlich-kommunikativen Phänomene sind zwei Faktorenbereiche kausal verantwortlich: die biologischen, bei denen sprachliche mit physischen Veränderungen korrelieren, die aber in dieser Arbeit unberücksichtigt bleiben, und die soziologischen Erklärungsmodelle. Hierbei sollen bestimmte sprachlichkommunikative Phänomene mit den strukturellen Veränderungen der sozialen Lebenssituation im Alter und mit den Veränderungen der sozialen Beziehungen zusammengebracht und aus ihnen hergeleitet werden.

Das Konzept der Lebenslage ist dabei insofern von Bedeutung, als dass verschiedene Lebenslagendimensionen im Hinblick auf ihren möglichen Einfluss auf sprachliche Veränderungen im Alter untersucht werden. In diese Untersuchung sollen folgende Dimensionen einbezogen werden: Wohnsituation, soziale Aktivität und Bildung.

Die methodische Vorgehensweise impliziert die Aufnahmen sprachlichen Datenmaterials anhand offener Interviews, die im Anschluss mithilfe einer Liste differierender Merkmale (Reinhard Fiehler) analysiert werden, und parallel dazu die Erstellung von Fragebögen zu den sozialen Faktoren bzw. Lebenslagendimensionen, die statistisch in Bezug auf das Sprachmaterial ausgewertet werden sollen. 


\section{Section III Language in Education}

\section{Jacqueline Thissen}

Kleine Wörtchen haben es in sich! Sind Partikeln im engeren Sinne doch ein Thema für die Schule?

Vor der so genannten pragmatischen Wende wurden Partikeln im engeren Sinne nur als periphere Elemente der Rhetorik angesehen und auf Grund ihrer primär kommunikativen Funktion in der gesprochenen Sprache bzw. ihrer nicht vorhandenen morphologischen und syntaktischen Eigenschaften von der sprachwissenschaftlichen Forschung nicht weiter beachtet, sodass sie sowohl in Grammatiken als auch in Wörterbüchern vernachlässigt wurden. Das zeigt sich beispielsweise daran, wie Partikeln auch von Wissenschaftlern vor der einsetzenden Partikelforschung charakterisiert wurden. „Alle diese Flickworte wimmeln wie Läuse in dem Pelz unserer Sprache herum." (Reiners 1943: 340). Mit dem Interesse der Sprachwissenschaft an kommunikativen und pragmatischen Funktionen erwuchs auch die Partikelforschung, sodass Partikeln nun seit mehreren Jahrzehnten in der Linguistik Beachtung gefunden haben und als Bestandteil in fast jeder Grammatik integriert sind.

Die Thematik der Partikeln wird in der Schule ausgeklammert. Weder kommen sie in Schulbüchern vor noch werden sie im Zusammenhang mit den zu behandelnden Wortarten in den Richtlinien und Lehrplänen aufgeführt, obwohl sich eine Auseinandersetzung mit Partikeln, betrachtet man die Ziele und Aufgabenbereiche der Richtlinien und Lehrpläne, durchaus eignet, um viele dieser Ziele zu erreichen. Z.B. die Sprache als Ergebnis eines ontogenetischen, historischen und interlingualen Entwicklungsprozess' zu sehen oder die Einschätzung der eigenen Sprache im Kontrast zu anderen. Darüber hinaus lassen sie sich ebenso mit den anderen Bereichen des Deutschunterrichtes wie Umgang mit Texten und Medien oder Sprechen verknüpfen.

In diesem Vortrag wird der Schwerpunkt auf die grammatische Betrachtung gelegt und gezeigt, welches Potential die grammatische Analysearbeit von Partikeln in der Schule in sich trägt.

Wenn man sich beispielsweise die Möglichkeit, Wortarten zu kategorisieren, bei Boettcher (2010) oder Sitta/Gallmann (2007) näher anschaut (Reihenfolge der Einteilung erfolgt nach Morphologie, Syntax, Semantik, Pragmatik), wird deutlich, dass Partikeln erst dann kategorisiert werden können, wenn bereits sämtliche Kriterien auf morphologischer, syntaktischer und semantischer Ebene erschlossen sind. Das bedeutet, dass die grammatische Analysearbeit als eine komplexe Gesamterfassung der Wortarteneinteilung anzusehen ist, bei der es unabdingbar ist, Schwarz-Weiß-Raster von Wortarteneinteilungen fallen zu lassen, da Partikeln als Grenzgänger nur vor dem Hintergrund der jeweiligen Kriterienentscheidung kategorisiert werden können. Weiterhin wird gezeigt, wie sich die Umsetzung grammatischer Analysen mit fachdidaktischen Konzeptionen zum Grammatikunterricht, z.B.: Menzels Grammatikwerkstatt (2009), legitimieren lässt. 


\section{Jan Jorissen}

\section{The Effect of Writing Assignments in an Academic Writing Programme}

Students starting at university will find themselves being introduced to a new community with rules and norms that are different, and often unknown. The community's discourse generates great expectations and challenges and it takes time to getting accustomed to it. The students have their own expectations of what becoming an academic means, but the literature is clear that acculturation entails learning to communicate in a way that is accepted by the community. At the University of Antwerp, first-year students of English take an introductory course called All Write, which is set out to teach freshmen how to acculturate in this new environment for higher learning, through teaching them the basics of academic or argumentative prose for different evaluative purposes: writing e-mails to professors, making summaries, doing outlines, writing exam papers, etc. A key question in my research is how students perceive the gradual change in their acculturation brought about by the course. To this end I will study how they evaluate the in-class assignments. Not only will I correlate the data from the 5 assignments evaluated during the first term, I will also look at how the students $(n=130)$ evaluated the final assignment and whether these are consistent with their overall evaluation. In doing so I hope to map the students' perception of the growth in their own academic writing on the wider process of acculturation.

\section{Carola Strobl}

Summary writing from aural input in a foreign language: How can this complex task be supported by an online learning module?

(How) can a complex task like writing a summary from aural input be effectively supported by an online learning environment? What are students' beliefs and attitudes towards this instructional medium? These are the two key issues in the presented pilot study for my doctoral thesis. Kirkland \& Saunders (1991) refer to the importance of mediating the "overwhelming cognitive overload" in summarizing tasks. My aim is to find out how an online learning module can facilitate this mediation by scaffolding the learners throughout the different steps of the process, from listening to building up the structures and vocabulary required in a coherent summary.

For this experimental study, 38 ba2 students in an applied linguistics programme followed an online learning module on summarizing aural input, which was created specifically for this target public, for 4 weeks. Having completed preparation exercises on content and language related aspects, they wrote a weekly summary of a 3-5 min radio feature in German. An important focus of the study lies on the students' attitudes towards instructivist vs. constructivist tasks. The module therefore contains various types of exercises, ranging from closed pattern-drill exercises with pre-programmed automatic feedback to open answer exercises with sample solutions for auto-correction.

For a progress analysis regarding coherence and cohesion, a pre- and an immediate post-test were used that consisted of two parts: a gap filling exercise on cohesion structures and linking words, and a summary of a radio feature on the same topic. A preliminary analysis of the summaries shows that approximately $40 \%$ of the students clearly benefited from the online learning module in the sense that their post-tests reveal a significant progress in at least one of the two major criteria. 
In order to shed light on the students' beliefs and attitudes towards this instructional medium which is relatively new to them, several short online surveys were carried out in the course of the experiment. The most interesting results in this respect were obtained through two focus group interviews at the end of the experiment. Some unexpected attitudes were revealed, such as a certain reluctance to use the internet for instructional purposes in addition to the daily hours spent online for private communication.

\section{References:}

Kirkland, M. R. \& Saunders, M. A. P. (1991): Maximizing Student Performance in Summary Writing: Managing Cognitive Load. TESOL Quarterly, Vol. 25, No. 1, pp. 105-121

\section{Deogratias Nizonkiza}

Relationship between lexical competence, collocational competence, and second language proficiency: The case of Belgian English majors

The present study replicates Nizonkiza (in press), which echoed research interests into vocabulary, in particular studies associating vocabulary with L2 proficiency (Meara and colleagues; Zareva et al. 2005; Nation \& Beglar 2007). Nizonkiza (in press) developed a test modelled on Read's (1993) Word Associates Test with a focus on collocations and administered it to English majors at the University of Burundi in order to answer the following research questions:

- To what extent can lexical competence predict L2 proficiency?

- To what extent can collocational competence predict lexical competence?

- To what extent can collocational competence predict L2 proficiency?

- Which collocations, of the more frequent and the less frequent, cause more difficulties for L2 learners?

On the basis of TOEFL and vocabulary test scores, Nizonkiza (in press) established a relationship between $\mathrm{L} 2$ proficiency, lexical competence, and receptive collocational competence. Following the view that culture and language are linked (Van de Poel \& Swanepoel 2003), his study was replicated on English majors at the University of Antwerp with Dutch as their L1 and it reached similar conclusions. The rationale of extending the study to learners with a different socio-linguistic background is that it might contribute to (i) making more reliable predictions about the major role played by vocabulary in language testing and (ii) conducting a qualitative analysis of the test items in order to improve the weak ones and then further the process of developing a more valid test. Results from the study strengthen recent efforts (Keshavarz \& Salimi 2007; Gyllstad 2007, 2009; Eyckmans 2009) to associate vocabulary with L2 proficiency.

\section{References}

Eyckmans, June

2009 Toward an assessment of learners' receptive and productive syntagmatic knowledge. In Researching Collocations in Another Language, Andy Barfield \& Henrik Gyllstad (eds.), 139-152. New York: Palgrave Macmillan. 
Gyllstad, Henrik

2007 Testing English collocations. Unpublished PhD Dissertation, Lund University.

Gyllstad, Henrik

2009 Designing and evaluating tests of receptive collocation knowledge: COLLEX and COLLMATCH. In Researching Collocations in Another Language, Andy Barfield \& Henrik Gyllstad (eds.), 153-170. New York: Palgrave Macmillan.

Keshavarz, Mohammad Hossein \& Hossein Salimi

2007 Collocational competence and cloze test performance: A study of Iranian EFL learners. International Journal of Applied Linguistics 17: 81-92.

Nation, Paul \& David Beglar

2007 A vocabulary size test. The Language Teacher 31: 9-13.

Read, John

1993 The development of a new measure of L2 vocabulary knowledge. Language Testing 10: 355-371.

Van de Poel, Kris \& Piet Swanepoel

2003 Theoretical and methodological pluralism in designing effective lexical support for CALL. CALL Journal 16: 173-221.

Wray, Alison

2002 Formulaic Language and the Lexicon. Cambridge: Cambridge University Press. Zareva, Alla, Paula Schwanenflugel \& Yordanka Nikolova

2005 Relationship between lexical competence and language proficiency. Studies in Second Language 27: 567-595.

\section{Jelena Danilović, Ivana Zorica, Branimir Jeremić}

Serbian, English and German in interaction: A study of cognates and German L3 learners in Serbia

The role of cognates in $\mathrm{L} 2$ acquisition has been extensively researched over the past twenty years yielding evidence that learners are continuously making links between the words they already know in one language and the target language words (Laufer, 1990; Ringbom, 2007) whereas research into cross-linguistic influence in L3 acquisition is still in its infancy (Cenoz, Hufeisen and Jessner, 2001). Even though Serbian is not closely related to German or English, its lexicon has been augmented by loanwords from Greek, Latin, French, German and English in the course of time due to political, economic or cultural circumstances. On the other hand, German and English share as many as 8,000 identical or similar lexemes according to certain estimates (Braun, 1979). The purpose of this study, therefore, was to explore the influence Serbian-German, English-German and Serbian-English-German cognate lexemes exert on beginner German L3 learners in Serbia, that is, to establish what the learners' awareness of cognates was. It stands to reason to hypothesize that cognates, especially Serbian-English-German ones, will facilitate the acquisition of related German lexemes. We intend to validate this assumption by means of a reading comprehension test devised after the manner of Nagy et al. (1992). Our results could have pedagogical implications for German L3 learning in Serbia, contributing to curriculum design and a better understanding of lexical development in L3 learners. 


\section{Dieuwerke Rutgers}

Metalinguistic Development in Context: Learning German as a Third Language within a bilingual education programme and a regular programme in the Netherlands

In an increasingly globalised world, foreign/second language learning has become a crucial part of people's identities as global citizens. In particular, the spread of English has led to the development of "multilingualism with English" and, consequently, to a growing interest in multilingualism and third language (L3) learning as an area of research. Research within this field has increasingly shown that bi/multilingualism can lead to benefits in relation to learning additional languages. This presentation will report on a Case Study conducted in the Netherlands with the aim of both exploring the applicability of L3 learning theories to foreign language pedagogy and supporting the teaching of foreign languages alongside English as a lingua franca. The study consists of a comparative analysis of the metalinguistic skills of pupils following a Dutch-English bilingual education programme and a regular programme, focussing particularly on how they use these skills when learning German as a third language. The study looks at L3 learning from a socio-cognitive and Sociocultural perspective (i.e. discursive activity about language (in social interaction) leads to knowledge of language that allows for its control, consequently impacting on and mediating the learning of further languages), and consisted of task-based research (recorded group-tasks) with restrospective interviews and classroom observations. Initial findings suggest that the metalinguistic skills of pupils following bilingual education are of a different nature to those of their regular counterparts, revealing a deeper understanding of language as a communicative and meaning-making tool which affects the way the pupils approach a task relating to the learning of German.

\section{Section IV Language in Professional Settings}

\section{Gregor Geiermann, Friederike Sell \\ "How to greet a professor?": identifying emerging e-mail etiquette in professor-student communication}

"Emerging e-mail etiquette" (EEE) is an on-going large-scale empirical research project at Bonn Applied English Linguistics (BAEL). It combines a wide range of methods from empirical pragmatics to experimental psycholinguistics, such as questionnaires, metapragmatic interviews, focus group discussions, monological and dialogical think-aloud protocols and key-logging text production processes. The current focus is on student e-mails to professors, specifically on "apologising" e-mails and "requesting" e-mails, and on what is considered appropriate in these contexts.

The key issue is how the writers position themselves vis-à-vis their addressees. Examining the issue involves analysing the linguistic choices made by the writers and the decisionmaking processes underlying these choices, and the judgement of appropriateness by other students and professors. Further questions dealt with the following: Which role does the gender of text producers and text receivers play? Do differences exist across languages and between e-mails written in the native language and e-mails written in a foreign language? How are relationships negotiated in e-mail sequences? Are there any differences across academic disciplines and faculties? Have practices changed over the past two decades? 
At its current stage the project investigates "requesting and apologizing" e-mails written in English and German over the past five years. The features highlighted include opening and closing routines, "apologising and requesting" strategies, reasons given (in apologising), detail, correctness, and emoticons. The data discussed are taken from a corpus of naturally occurring e-mails, from production tasks and from rating tasks. In the nearer future, the project infrastructure will migrate to a database-driven web-application, seeking to harmonise the different research methods applied to the e-mail corpus.

\section{Inge Blockmans}

Accommodation in Special Needs Communication; Disabled Student's. Perception of Communication about Personal Functional Problems

Consciously or not, individuals adjust their communicative behaviour on the basis of 'judgments of their conversational partners' communicative characteristics', their own 'desire to establish and maintain a positive personal and social identity', the specific purpose of the interaction, and the context in which the interaction takes place (Gallois et al. 2005). What makes interaction especially challenging is the fact that speakers may accommodate to stereotypical rather than to truly individual features of others, which is a categorisation process that may severely restrict the relationship between interactants.

Communication Accommodation Theory - considered as 'one of the best-developed theories relating to interpersonal adjustment' (Littlejohn 1999: 107) - provided the framework for analysing disability disclosure and communication about special needs as experienced by seven disabled university students in Antwerp. Both quantitative and qualitative data were obtained by means of a questionnaire (multiple-choice questions and statements evaluated on a 5-point Likert scale) and a semi-structured interview.

The main questions guiding this study are: (1) Do students with visible physical disabilities experience shortcomings in communication accommodation in their professional environment?, and (2) Do they experience differences in communication depending on the interactant (coordinator of special facilities vs. professors vs. peers)?

First, the data will be examined along the possible levels of 'mis'accommodation, i.e. the appropriateness of the accommodation regarding the interactants' social role or status and the contextual situation (in or outside of class, etc.); the extent of engagement in the conversation; the terminology used to refer to 'disability'; the speaker's mood or emotion; topic management. Secondly, it will be analysed how interpersonal control strategies are affected and to which extent. The study will throw light on the roles which the interactants enact themselves (through the eyes of the disabled respondents), how the roles change due to 'mis'accommodation, and what the implications of role-shifting are for the interactants' future relationship. 


\section{Almut Schön, M.A.}

Arzt-Patienten-Interaktion und MS-NMS-Kommunikation: Gespräche ausländischer Ärzte mit deutschen Patienten aus diskursanalytischer Sicht

In deutschen Krankenhäusern arbeiten zunehmend Ärzte und Ärztinnen, die Deutsch als Zweitsprache benutzen (L2-Sprecher). Im Rahmen einer Kooperation zwischen der Charité Universitätsmedizin und der TU Berlin, Fachgebiet Deutsch als Fremdsprache wurden Anamnesegespräche dieser Ärzte mit deutschen Patienten aufgezeichnet. In meiner Dissertation werden diese Gespräche transkribiert und mit dem Ziel analysiert, die Architektur des Verstehens in diesem Diskurs zu beschreiben.

Dabei ist meine methodologische Hypothese, dass sich gerade die vielfältigen, sich überlagernden Aussagenfelder in diesem Diskurs adäquat mit den Methoden der Diskurslinguistik nach Foucault (s. Warnke \& Spitzmüller 2008) beschreiben lassen, auch wenn Gespräche in diesem Rahmen bisher eher wenig erforscht sind. Dieses diskursanalytische Vorgehen berücksichtigt sowohl die Akteure, das Wissen der Akteure und die Sprache der Akteure (ibid.:17) und fragt dabei nach den Ermöglichungszusammenhängen (Diaz-Bone 2006) bestimmter Aussagen.

Im Fall des vorliegenden Korpus bedeutet dieses Vorgehen, alle Transkriptionen auf drei, einander überlagernden und durchdringenden Ebenen zu codieren:

- nach dem Fragenkatalog der Anamnese

- nach den Sprechhandlungen wie Protest, Zustimmung, Ablehnung, Entschuldigung etc.

- nach Gesprächsphänomenen, darunter auch solchen, die für Gespräche zwischen MS und NMS typisch sind, wie Bedeutungsaushandlungen, Code-Switchings etc.

An konkreten Beispielen soll im Vortrag die Arbeit mit diesen Codes gezeigt werden und insbesondere darauf eingegangen werden, wie diese Codierung dazu beitragen kann, die komplexe Architektur des Verstehens nachzuzeichnen und den Diskurs mit einem poststrukturalistischen Ansatz zu beschreiben.

\section{Literatur:}

Diaz-Bone, Rainer (2006): Zur Methodologisierung der Foucaultschen Diskursanalyse. In: Forum: Qualitative Sozialforschung, Jg. 7, H. 1. Online verfügbar unter http://www.qualitative-research.net/index.php/fqs/article/view/71/145.

Warnke, Ingo H.; Spitzmüller, Jürgen (Hg.) (2008): Methoden der Diskurslinguistik. Sprachwissenschaftliche Zugänge zur transtextuellen Ebene. Berlin: de Gruyter.

\section{Donata Lisaité}

Mobile Medical Professionals: Transcultural Communication Problems on the Workfloor

The dynamics of globalization are 'one of the key forces of social transformation in the contemporary world' (Castles 2002). Social transformation can be closely observed in the patterns of professional mobility: in recent years this mobility is taking on new meanings given that more and more people, as a result of developments in information and transport technology, are living their lives in two or more countries rather than settling in one of them. As a result of these temporary migration movements, communication patterns change and there arises a need to think in terms of transcultural communication. Thurlow (2003) explains that the term 'transcultural' is the most accurate to define the changing world. The 
term expresses 'the conceptual spaces that open up between traditionally defined cultural systems' and the fluidity among them. Also, the term covers the implications and controversies that emerge because of 'shifting patterns of sociocultural organisation and practice'.

This can also be seen in the internationalization of medical contexts. Recent research (Lisaite 2010) has shown that although most mobile medical professionals claim that their professional functioning within new cultural environments was very satisfying, they encountered difficulties on other, more personal levels of communication and failed to build personal relationships with colleagues or with people outside work. This reflects Templer's (2006) observation that while living and working abroad interaction adjustment (as opposed to general and work adjustment) is most difficult to achieve. In fact, Lisaitè (2010) shows that even though mobile medical professionals' personal lives may seem to be disconnected from their professional discourse, they influence performance on the workfloor to such a great extent that sometimes they can become the main reason for why professionals' careers abroad do not last. Thus, medical the professionals' motivation to stay abroad was shown to be greatly influenced by their transcultural communication skills: successful interaction adjustment correlated highly with willingness to stay and work abroad. The opposite was shown to be true as well: the lower the interaction adjustment skills, the lower the motivation to stay abroad.

The inadequacy of interaction adjustment consists of gaps within transcultural communication skills. In order to find some possible solutions for ensuring smoother transcultural communication it is crucial to pinpoint what nature (linguistic, cultural, social, personal or a combination of some/all) these inadequacies have and where exactly they lie. Identifying and classifying them will shed new light on what hinders successful integration of mobile medical professionals within a new society and new work settings. In this study, the focus is on the comparison between the perceptions by mobile medical professionals of communication problems that they experience on the workfloor and the problems that they have as observed by their local colleagues. Some preliminary findings of the on-going research will be presented at this conference.

\section{Verena Warda}

\section{The Motivation of Teachers of EFL in Teacher Training in NRW}

Teacher training in Germany, and specifically in North Rhine-Westphalia (NRW), is characterized by a high degree of voluntary participation. Teachers are legally obliged to participate in professional development courses but there is no obligation as to the amount of training or the content of training. Then again, standards for professional teachers have been defined by the Standing Conference of the Ministers of Culture and Education (Kultusministerkonferenz) already in 2004. These standards are quite detailed and very specific as to the skills a teacher should dispose of today.

Looking at concepts describing motivation of employees in the business world, the question is in how far these can be transferred to the educational world.

My research aims at the investigation of this perceived gap of theoretical concepts for teacher motivation. The focus will be on an analysis of the of current EFL teacher training courses on offer for teachers and on the other hand I will investigate the individual EFL teachers' motivation for and interest in his/her professional development. My paper will present initial findings from interviews conducted with German EFL teachers on the topic. 
